Originalveröffentlichung in: Texte zum Rechts- und Wirtschaftsleben (Texte aus der Umwelt der

Alten Testaments, Neue Folge 1), Gütersloh 2004, S. 185-207

\title{
Rechtskodex von Hermupolis (P. Kairo JE 89.127-30+89.137-43)
}

\section{Martin Andreas Stadler}

Der sogenannte Rechtskodex von Hermupolis wurde bei den Grabungen im mittelägyptischen Hermupolis, dem heutigen al-Aschmunein, 1938-39 entdeckt. Die teilweise schlecht erhaltene Papyrusrolle ist auf beiden Seiten beschrieben: Das verso enthält mathematische Übungen, während das recto mit einem juristischen Text in demotischer Schrift beschrieben wurde. Dieser Text ist der ohne Zweifel bedeutendste ägyptische Rechtstext, der bislang bekannt ist und nicht wie Urkunden und Gerichtsprotokolle die praktische Umsetzung ägyptischen Rechts dokumentiert, sondern das ägyptische Recht als Konzept für mögliche Fälle kasuistisch entwickelt. Zwar sind andere demotische Rechtsbücher bekannt, doch diese sind sehr fragmentarisch erhalten. Die Handschrift stammt aus der ersten Hälfte des 3. Jh. v. Chr., ist aber eine Sammlung älterer Texte, die mindestens teilweise nicht jünger als die Zeit vor Alexander d. Gr. sind, sogar aber bis in das 8. Jh. v. Chr. zurückgehen könnten. D.h. der Rechtskodex von Hermupolis überliefert rein ägyptisches Recht, wie es vor der makedonisch-ptolemäischen und sogar persischen Herrschaft praktiziert wurde. Die Sammlung selber, von der der Hermupolis-Kodex eine Abschrift ist, wurde wahrscheinlich im 6. Jh. v. Chr. angelegt, entweder während der 26. Dynastie oder während der ersten persischen Besatzung - nach Diodor I 95 war Darius I. einer der Gesetzgeber Ägyptens und gab dem verso des demotischen Papyrus Bibliothèque nationale 215 (wohl aus dem 3. Jh. v. Chr.) zufolge 519 v. Chr. den Befehl, die ägyptischen Gesetze zu sammeln. Neben kalendarischen Erwägungen führen außerdem sprachliche Archaismen zu dieser Datierung. Ein Fragment einer griechischen Übersetzung ist aus dem 2. Jh. n. Chr. überliefert, das aber wiederum eine Abschrift eines frühptolemäischen Textes ist, von dem seinerseits vermutet wird, es basiere auf einer anderen, verlorenen Abschrift des Rechtskodex. Die Textüberlieferung muß also mindestens von einer Urversion und zwei Abschriften ausgehen, von denen eine die Vorlage für die griechische Übersetzung in frühptolemäischer Zeit war, die uns in einer fragmentierten Abschrift aus römischer Zeit vorliegt. Die andere Abschrift der Urversion wiederum ist entweder der Codex Hermupolis selbst oder eine Vorlage dessen.

Die erste und die letzte erhaltene Kolumne des Papyrus sind stark zerstört - nur noch die Zeilenenden der ersten und die Zeilenanfänge der letzten erhaltenen Kolumne sind noch vorhanden -, inhaltlich kaum noch $\mathrm{zu}$ fassen und deshalb hier nicht wiedergegeben worden. K. Donker van Heel legte die letzte vollständige Übersetzung des Textes vor, wobei er auch unpublizierte Transliterationen und Übersetzungen von M. Malinine und P. W. Pestman heranziehen konnte. Letzterer hat den Originalpapyrus in Kairo untersucht. Es scheint aber die Arbeit von K. Donker van Heel eher eine Kompilation bestehender Transliterationen, Übersetzungen und Kurzbemerkungen als eine eigenständige Bearbeitung des Papyrus zu sein, wie z.B. die ins Englische und Französische übersetzten Abschnitte, die einfach nebeneinander stehen blieben, die Klammersetzungen und die Fehlstellen in Abweichung von den publizierten Pho- 
tos des Papyrus anzeigen oder auch manche ungenauen Wiedergaben des Demotischen belegen, die aus älteren Arbeiten übernommen worden sind.

Die Bezeichnung »Rechtskodex« hat sich eingebürgert, während auch die Bezeichnung »Rechtshandbuch« (»legal manual«) von Pestman für den Rechtskodex von Hermupolis eingeführt worden ist, weil seiner Meinung nach eher Erläuterungen zur Ausführung als eine reine Gesetzessammlung gegeben würden. Die Abschnitte des Rechtskodex werden durch Spatien abgesetzte Überschriften gekennzeichnet und beschäftigen sich mit dem Pachtrecht, das sich aus Ackerrecht, Formen der Beschwerde und dem Mietrecht zusammensetzt, mit dem Unterhaltsrecht, dem Baurecht, dem Erbrecht und zuletzt dem Grabrecht.

Durch seine äußere Gestaltung gliederte der Schreiber des Rechtskodex von Hermupolis den Text in verschiedene Kapitel und Abschnitte, indem er Phrasen oder Formeln also durch Spatien vom Rest des Textes absetzte. Mit diesen Spatien werden so die einzigen erhaltenen Kapitelüberschriften »Recht über die Pacht " $(x+I I, 23)$ und "Dotationsrecht $(x+I V, 6)$ hervorgehoben. Die innerhalb der Kapitel weiterhin gesetzten Spatien markieren die Formeln, die einen Mustertext für Urkunden, Quittungen oder Eide einführt, z. B.: »Die Dotationsurkunde, die man ausstellt. Ihr Wortlaut: (...) «Der folgende Wortlaut wird ebenfalls von Spatien eingerahmt.

Doch damit ist die Verwendung von Spatien im Rechtskodex von Hermupolis noch nicht in sämtlichen Funktionen erläutert. Es werden nämlich nicht nur Mustertexte gegeben, sondern auch möglichst alle denkbaren Streitfälle ausgeführt, wie sich ein Kläger gegen einen Beklagten wendet und wie der Beklagte sich zu rechtfertigen versuchen könnte. Vor jedem dieser Fallbeispiele wird ebenso ein Spatium gesetzt.

Aus der Beschreibung der äußeren Gestaltung werden die verschiedenen inhaltlichen Ebenen und auch die Gliederung der Handschrift deutlich. Der erste erhaltene Themenbereich, zu dem die ägyptische Überschrift jedoch verloren ist, ist das Pachtrecht. Dabei muß es sich um ein bestimmtes Teilgebiet handeln, weil in $\mathrm{x}+\mathrm{II}, 23$ die Kapitelüberschrift »Recht über die Pacht « das eigentliche Pachtrecht einleitet. Welches Teilgebiet dies ist, ist schwer zu bestimmen, denn sowohl vor als auch nach der Überschrift »Recht über die Pacht« werden Probleme aus der Ackerpacht und der Hausvermietung diskutiert. Im ersten Themenbereich dreht sich das Hauptaugenmerk um die Verpachtung von Ackerboden und den damit verbundenen Pachtzins, doch auch der Verkauf eines Hauses wird thematisiert. Bei diesem Fall soll ein Haus verkauft werden, das allerdings der Verkäufer einem Schuldner als Pfand gegeben hat. Um zu verhindern, daß sich der Schuldiger durch den Hausverkauf seinen Verpflichtungen entzieht, muß der Schuldner 3 Jahre lang dagegen öffentlich protestieren, um zu verhindern, daß der Käufer freien Anspruch auf das Haus erheben kann.

Zur Feststellung, ob ein Haus irgendwie belastet ist oder nicht, verpachtet der Käufer das Haus an einen Dritten. Wenn nun drei Jahre lang kein öffentlicher Protest um dieses Haus erhoben wurde, kann der Käufer sicher sein, daß er die vollen Eigentumsrechte an dem Haus hat, das nicht etwa an eine andere Person verpfändet ist. Das ist das erste Problem, mit dem sich das Kapitel befaßt, zu dem uns der ägyptische Titel »Recht über die Pacht» $(\mathrm{x}+\mathrm{II}, 23)$ erhalten ist, um dann einen ähnlichen Fall für einen Acker und die Vermietung gewerblicher Immobilien, die dazugehörigen Urkundentexte und etwaige auftretende Säumigkeiten bei der Ausbezahlung der Miete zu be- 
handeln. Es ist zu beachten, daß der Pächter dem Verpächter eine Urkunde ausstellt, was das Ägyptische mit »einem über etwas schreiben« ausdrückt. Das Kapitel schließt mit einem Mustertext zum "öffentlichen Protest", der vom Verpächter erhoben wird bei ausbleibenden Zahlungen der Miete, sowie der Vorlage, wie eine Quittung für Mietzahlungen zu formulieren sei.

Ein wesentlicher Teil des erhaltenen Papyrus behandelt das sogenannte »Dotationsrecht « ( $h p n$ s $n h$ ). Die Übersetzung »Dotationsrecht « ist freilich nicht unproblematisch, wie unten noch ausgeführt werden wird. Eine vergleichbare Konzeption, bei der zwei Parteien A und B zugunsten einer dritten Partei C vereinbaren, daß A an B Geld - die Dotation $\left(s^{c} n h\right)$ - zahlt, damit B für den Unterhalt von C aufkommt, ist im abendländischen Recht nicht bekannt. Diese eigentümliche Praxis fand offenbar auch im ptolemäischen Ägypten dann keine Anwendung mehr. Alle damit verbundenen und möglicherweise auftretenden Unregelmäßigkeiten in der Form, daß die eine oder andere Partei ihren Verpflichtungen nicht nachkommt, werden mit den entsprechenden Verfahrensregeln erläutert, ebenso wie der Wortlaut für etwaige Quittungen gegeben wird. Auch die Möglichkeit des Todesfalles einer der Parteien wird diskutiert bis hin zu dem Fall, daß behauptet wird, es werde für eine bereits verstorbene Frau Unterhalt gefordert.

Zwischen den Kolumnen $\mathrm{x}+\mathrm{V}$ und $\mathrm{x}+\mathrm{VI}$ fehlt vermutlich mindestens eine weitere Kolumne, denn inhaltlich ist der Themenkomplex des Dotationsrechtes abgeschlossen, und ab x+VI stehen Fragen des Eigentums an Immobilien im Mittelpunkt. Die Überschrift ist allerdings heute verloren. Der erste Fall ist nicht mehr vollständig erhalten, aber offenbar lehnen es zwei Parteien ab, sich mit rechtlichen Altlasten eines Hauses zu befassen, die von zwei anderen Menschen zu verantworten sind. Außerdem sind Streitfälle aufgeführt, bei denen ein Haus von jemandem auf einem Grundstück errichtet wird, auf das ein anderer Ansprüche erhebt, weil er es von seinem Vater ererbt habe, oder ein Haus wird von zwei Parteien als ererbt oder rechtmäßig erworben betrachtet. Neben Streitigkeiten über ausstehende Zahlungen im Rahmen von Hausverkäufen kommen auch baurechtliche Probleme zur Sprache: Wie ist zu verfahren, wenn einer ein Haus baut und dabei das Haus eines anderen verbaut? Wie sind Schadensersatzforderungen in diesem Zusammenhang zu behandeln? Wie ist zwischen Nachbarn eine Entscheidung zu finden, die sich über unter Umständen unzulässig okkupierten Grund, das Verbauen von Zugängen oder Beeinträchtigungen der eigenen Immobilien durch den nachbarlichen Neubau streiten?

Gegen Ende des erhaltenen Papyrus (x+VIII, 30) wird noch das Erbrecht thematisiert, insbesondere Streit zwischen den Nachkommen eines Erblassers. Dabei geht es um Erbanteile. Da der älteste Sohn die Anteile seiner vor dem Erbfall kinderlos verstorbenen Brüder erhält, können seitens der anderen Erbnehmer Zweifel aufkommen, ob alle vom ältesten Bruder genannten und verstorbenen Personen tatsächlich Kinder des Erblassers sind. Auch für diese Möglichkeit wird das entsprechende Verfahren ausgeführt. Oder es wird angefochten, daß ein jüngerer Sohn schon zu Lebzeiten des Vaters Besitz erhalten hat, der dann nicht mehr Teil der zu verteilenden Erbmasse ist.

Nachdem Regelungen für Töchter - sie sind gegenüber Brüdern benachteiligt bzw. die älteste Tochter bekommt nicht die Anteile ihrer verstorbenen Schwestern, wenn der Erblasser nur Töchter hatte - und auch für den Hausverkauf durch den ältesten 
erbenden Sohn und die sich daraus ergebenden Ansprüche der Brüder aus dem Erlös des Hausverkaufs getroffen wurden, findet noch kurz das Grabrecht Erwähnung: Ein Grab darf nur dann frei verkauft werden, wenn es noch nicht belegt wurde. Danach bricht der Papyrus, von wenigen Schriftresten der letzten Kolumne abgesehen, ab. Wieviele Kolumnen ehemals dann noch folgten, kann nicht mehr bestimmt werden.

In der hier vorgelegten Neuübersetzung ist versucht worden - soweit die Verständlichkeit nicht beeinträchtigt wird -, so nah wie möglich am ägyptischen Original zu bleiben, um nicht durch falsche Wahl moderner juristischer Termini seitens eines juristischen Laien, der der Übersetzer ist, die ägyptischen juristischen Fachbegriffe zu verfälschen. Ferner decken sich die Begrifflichkeiten zweier so unterschiedlicher Rechtskulturen wie die der altägyptischen und der abendländischen in den seltensten Fällen, wie etwa das Problem des ägyptischen Wortes $n b »$ Herr« exemplifizieren mag, das weder mit dem deutschen »Besitzer« noch »Eigentümer« korrekt wiedergegeben ist. In diesem Falle ist daher die Grundbedeutung des ägyptischen Wortes »Herr « in dem Sinne beibehalten worden, daß hier ein neutraler Begriff zur Bezeichnung einer Possessivbeziehung zu einem Objekt gewählt wurde, der sowohl die Bedeutungen »Eigentümer« als auch »Besitzer« sowie »Inhaber« kommuniziert. Ähnliches gilt für $s^{i} n h$, das sich von »leben lassen « herleitet. Auf den ersten Blick mag »Unterhalt" eine angemessene Wiedergabe sein, zumal von $h p n s^{i} n h$ wörtlich also »Recht über das Lebenlassen « die Rede ist, innerhalb dessen dann Regelungen zu Unterhaltszahlungen getroffen werden. Doch trifft das den Bedeutungskomplex des ägyptischen $s^{e} n h$ nicht, zumal der eigentlich gezahlte Unterhalt ' $q$ - $h b s$ wörtlich »Brot und Kleidung" heißt, denn mit s'nh wird wie oben bereits erläutert ein Geflecht von Beziehungen zwischen drei Parteien zum Ziele des Unterhalts einer Person bezeichnet. Hier wurde nun $s^{c} n h$ mit $»$ Dotation « übersetzt - sicherlich nicht vollkommen befriedigend, zumal in der Grundbedeutung des ägyptischen Wortes nicht im entferntesten die Wurzel »geben« steckt.

Die Absätze der hiesigen neuen Übersetzung entsprechen den Spatien des demotischen Textes. Es ist also versucht worden, die äußere Gliederung der demotischen Handschrift zu spiegeln. Fettdruck kennzeichnet Hauptüberschriften, die ein neues Kapitel einleiten. Unterstrichenes sind Einleitungsformeln vor Mustertexten für Urkunden. Diese Hervorhebungen finden kein Gegenstück im Original etwa durch Rubrizierung, denn beides wird im Demotischen schwarz geschrieben, jedoch durch Spatien davor und auch danach abgesetzt. Die Verlustangaben in $\mathrm{cm}$ beziehen sich auf die geschätzte Länge des verlorenen und nicht mehr rekonstruierbaren demotischen Textes.

Literatur: Editio Princeps: G. Mattha, The Demotic Legal Code of Hermopolis West. Preface, additional notes and glossary by G. R. Hughes, BdE 45, Le Caire 1975. Deutsche Übersetzung: S. Grunert, Der Kodex Hermopolis und ausgewählte private Rechtsurkunden aus dem ptolemäischen Ägypten, Leipzig 1982. Vgl. zu dieser Publikation die Bewertung von P. W. Pestman, Remarks on the Legal Manual of Hermopolis: A Review-Article, in: Enchoria 12 (1984) 33-42. Letzte vollständige Transliteration und Ubersetzung: K. Donker van Heel, The Legal Manual of Hermopolis (P. Mattha). Text and Translation, Leiden 1990. Bibliographie bis 1985 bei S. Allam, Réflexions sur le »Code légal« d'Hermopolis dans l'Egypte ancienne, in: CEg 61 (1985) 50-75. Zur Datierung zusammenfassend: J. H. Johnson, The Persians and the 
Continuity of Egyptian Culture, in: H. Sancisi-Weerdenburg/A. Kuhrt/M. Cool Root (ed.), Continuity and Change. Achaemenid History VIII, Leiden 1994, 149-159. Zur griechischen Übersetzung: P. W. Pestman, Le manuel de droit égyptien de Hermoupolis. Les passages transmis en démotique et en grec, in: P. W. Pestman (éd.), Textes et études de papyrologie grecque, démotique et copte. P. L. Bat. 23, Leiden 1985, 116-143. Letzte Studie zu einem Einzelproblem mit zahlreicher weiterführender Literatur: A. Azzoni/S. L. Lippert, An Achaemenid Loanword in the Legal Code of Hermopolis: 3 bykrm, in: Enchoria 26 (2000) 20-30. Publikation des verso: R. A. Parker, Demotic Mathematical Papyri. Brown Egyptological Studies 7, London 1972, 13-53.

Kommentierte bibliographische Angaben zu weiteren juristischen Texten Ägyptens auf Demotisch bei: M. Depauw, A Companion to Demotic Studies. Papyrologica Bruxellensia 28, Bruxelles 1997, 113-115. Zu Quellen zur praktischen Anwendung s. dort auch auf Seite 139148.

$(\mathbf{x + I I}, \mathbf{1})$ Das Saatkorn: Bezüglich des Menschen, in dessen Hand das Saatgetreide ist, man läßt ihn die Äcker bestellen, deren Saatkorn in seiner Hand ist, und man läßt ihn über sie eine Pachturkunde ausstellen entsprechend ihrer ${ }^{1)}$ Art vom Vorjahr $\left.{ }^{2}\right),(\mathbf{x}+11,2)$ Der, der einen Acker bestellt, (und) [der], für den die Pachturkunde ausgestellt wurde: (Problem:) Wenn es ein Jahr ist, das ........ $[\ldots]^{3)}$

(Fall 1) Es gibt eine Pachturkunde, und sie ist gemacht, um ein Feld zu bestellen. Strafe für Vertragsbruch.4) Der Herr5) (x+II, 3) der Äcker gibt nicht Saatkorn für die nämlichen Äcker. Nur die Ernteabgabe der nämlichen Äcker gemäß der Pachturkunde, welche [ausgestellt wird], wird gegeben.

(Fall 2) Es gibt die Äcker der Leute, welche eine Pachturkunde ausstellen, um Äcker zu bestellen, und den, $[\mathrm{der}](\mathbf{x}+\mathbf{I I}, 4)$ die nämlichen Äcker bestellen [will]6), macht einen $\gg P a-$ pyrus über Gewinn «7) für die Leute, um ihnen eine Pachturkunde auszustellen, um die Äcker zu bestellen. [Man] zwingt [sie .................... mit ihnen für ein weiteres Jahr, bis sie das Saatgut (zurück)geben, das [in ihren Händen ist.]

(x+II, 5) (Fall 3) Wenn ein Mensch Felder bestellt, und der, der sie bestellt hat, für sie sorgt, und der Herr der Äcker sie von ihm nimmt, [sagend:] »»lch werde nicht von ihm eine Ernteabgabe verlangen. « (Und) wenn [der, der die Äcker bestellt hat,] $(\mathbf{x + l l ,}, \mathbf{6}) \mathrm{kla}$ gen wird gegen den Herren der Äcker, wird das Viertel des Ernteertrages genommen

1. Des Landbesitzers und Pächters.

2. K. Donker van Heel (in: $\mathrm{aaO} 11$ ) liest $p 3 y=w$ smt $n m h$ wtheir (...) free manner «. Zum Wort snf "Vorjahr "s. K.-Th. Zauzich, in: Enchoria 9 (1979) 145. Anwendung auf den vorliegenden Text: J. F. Quack, in: Enchoria 25 (1999) 47.

3. Vermutlich ist ein für die Bauern ungünstig verlaufendes Jahr gemeint.

4. $\mathrm{Zu} 3$ by krm als achämenidischem Lehnwort: A. Azzoni/S. L. Lippert, in: Enchoria $26(2000)$ 20-30.

5. Das ägyptische $n b$ bezeichnet "Herr, Besitzer, Eigentümer«. Eine Wiedergabe mit "Besitzer» oder "Eigentümer« wäre konkreter als der ägyptische Begriff und ist daher hier vermieden worden. S. auch: S. Grunert, Zur Frage eines Ersitzungs-Rechtes im ptolemäischen Ägypten, und ders., Zur Definition »Eigentum», in: S. Allam (Hg.), Grund und Boden in Altägypten, Tübingen 1994, 319-325.393f.

6. K. Donker van Heel (in: aaO 11) ergänzt hier statt $p_{3}[n t m r]$ sk ${ }_{3}$ wie hier vorgeschlagen $p_{3}^{3}$ $[n t i w=f(r)] s k_{3} \gg$ der, [der] pflügen [wird] $\ll$.

7. Zum problematischen Begriff: A. Azzoni/S. L. Lippert, in: Enchoria 26 (2000) 28. 
werden, welcher dem nämlichen Saatkorn entspricht, von dem Herrn der Äcker. [Es wird gegeben werden] dem, der die Äcker bestellt hat zum Lohn seiner Arbeit.

(Fall 4) Wenn $(\mathbf{x}+11,7)$ der Mensch, der die Äcker bestellt hat, nicht für sie sorgt, indem (nämlich) der Mensch, der sie bestellt hat, mit Saatgut von einem anderen bestellt: Wenn der Herr der Äcker die Äcker wegnimmt und der, der sie bestellt hat, klagt $(\mathbf{x}+11, \mathbf{8})$ gegen den Herren der Äcker, wird das Viertel des Ernteertrages durch den Herren der Äcker genommen werden, welches für das nämliche Saatkorn steht. Es wird dem gegeben werden, der die Äcker bestellt hat, als der Lohn seiner Arbeit und seines Saatkorns ${ }^{8)}$.

$(\mathbf{x + I I , 9 )}$ (Fall 5) Wenn ein Mensch eine Pachturkunde über die Äcker ausstellt, und der Herr der Äcker ihm Saatkorn gibt, und der Mensch, der eine Pachturkunde ausgestellt hat, nicht die Äcker bestellt, und er das Saatkorn nimmt, nachdem die Äcker überschwemmt wurden, $(\mathbf{x}+\mathbf{+ 1}, \mathbf{1 0})$ indem sie brach liegen'), man läßt ihn die Ernteabgabe des Saatkorns gemäß der Pachturkunde geben, die er ausgestellt hat.

Wenn ein Mensch eine Pachturkunde ausstellt über einen Acker, um ihn zu bestellen, und der Herr der Äcker ihm Saatkorn gibt, $(\mathbf{x}+\mathbf{l 1}, \mathbf{1 1})$ und die Äcker nicht überschwemmt werden, indem das nämliche Jahr ohne Überschwemmung ist, läßt man ihn keine Ernteabgabe geben. Man läßt inn das Saatgut (zurück)geben.

$(\mathbf{x}+\mathbf{I 1}, \mathbf{1 2})$ Siehe die Art, einen öffentlichen Protest zu verfassen, der gemacht werden wird wegen eines Hauses, das nicht (juristisch) rein war für den Menschen, dem es verkauft wurde:

$(\mathbf{x + l I}, \mathbf{1 3})$ »Regierungsjahr $\mathrm{X}$, Monat $\mathrm{Y}$. A, macht einen öffentlichen Protest gegen B: `Er hat veranlaßt, daß dem $C$ geschrieben werde bezüglich seines (des B) Hauses, welches im Ort soundso ist.« Und er beschreibt seine südlichen, (x+II, 14) nördlichen, östlichen und westlichen Nachbarn. »Dieses Haus, dessen Nachbarn oben beschrieben sind, er (B) hat mir (A) darüber als ein Pfand geschrieben. Er schuldet mir soundso viel Geld für $X$ Jahre, $Y$ Monate. ${ }^{10)}$ Über dieses $(\mathbf{x}+11, \mathbf{1 5})$ obige Haus hat er $(B)$ an $C$ geschrieben, indem er nichts (zu tun hat) mit obigem Haus, bis er mich vollständig mit meinem Geld, über welches er mir geschrieben hat, zurückbezahlt hat. $C$ hat nichts zu tun mit ihm (B) $(\mathbf{x}+\mathbf{I 1}, \mathbf{1 6})$ in bezug auf das Haus, bis er (B) mir soundso viel Geld vergolten hat, über das er mir geschrieben hat. Wenn er (B) nicht das Geld gibt, veranlasse, daß er fern sei von diesem obigen Haus. Ich habe ihn (B) nicht gefunden, um einen öffentlichen Protest zu erheben gegen ihn. (x+II, 17) Mein öffentlicher Protest für jedes Jahr ist es. « Wenn er (A) 3 Jahre verbringt, indem er jährlich einen öffentlichen Protest erhebt 〈für $\rangle$ den Besitz, weswegen drei öffentliche Proteste erhoben werden gemäß dem, was oben geschrieben ist, dann kann der Mensch, der das Haus ergreift, nicht $(\mathbf{x}+\mathbf{I 1}, \mathbf{1 8})$ sagen: $\gg$ Mein Haus

8. Der Pächter muß sich also mit demselben Betrag zufrieden geben wie im vorhergehenden Fall, obwohl er wegen des fremden Saatgutes einen höheren Aufwand hatte als dort - er hat sich ja auch nicht ordnungsgemäß um den Acker gekümmert. A. Azzoni/S. L. Lippert (in: Enchoria 26 [2000] 29) dagegen interpretieren irm $t_{5} y=f$ pr.t-sh.t als einen zum Viertel des Ernteertrages, der der Arbeitslohn ist, zusätzlichen Anspruch des Pächters. Das wird vom Demotischen nicht unterstützt, weil $t_{3}$ s šb.t "Lohn « sich auf $t_{3} y=f$ wp.t irm $t_{5} y=f$ pr.t-sh.t »seine Arbeit und sein Saatkorn« beziehen muß (so auch K. Donker van Heel, aaO 13).

9. S. A. Azzoni/S. L. Lippert, in: Enchoria 26 (2000) 29 Anm. 51.

10. D. h. B schuldet A eine bestimmte Summe Geldes. 
ist es. Es ist für mich (juristisch) rein.« Dieser öffentliche Protest, man erhebt ihn bezüglich allem, was man verkaufen wird, um zu verhindern, daß es rein ist 〈für) den, dem es verkauft wurde. $(\mathbf{x}+\mathbf{+ 1}, \mathbf{1 9})$ Wenn ein Mensch einen öffentlichen Protest in Anwesenheit erhebt, vor Zeugen macht er es, welche darüber niederschreiben, während der Mensch, gegen den der öffentliche Protest erhoben wird, anwesend ist.

Siehe den Wortlaut eines öffentlichen Protests in Anwesenheit, den ( $\mathbf{x + 1 1 , 2 0 )}$ man erheben wird:

»Regierungsjahr X, Monat Y. A erhebt gegen C, welcher anwesend ist, einen öffentlichen Protest: >Du (C) hast B für dich schreiben lassen bezüglich seines (des B) (x+11, 21) Hauses< - oder >Besitzes/oder >er hat genommen den soundso Besitz von mir/oder >von ihm « gemäß dem obigen öffentlichen Protest. Jedes Wort, das der Mensch sagen wird, man nimmt es in den öffentlichen Protest auf: $\gg C(\mathbf{x}+\mathbf{l 1}, \mathbf{2 2})$ ist anwesend, indem er das und das sagt.« Wenn er (C) nicht spricht, dann schreibt man in den öffentlichen Protest: »C ist anwesend, indem er schweigt und nicht spricht. «

(x+II, 23) Recht über die Pacht: Wenn ein Mensch sie macht wegen eines Hauses oder Besitzes.

Der Mensch (A), der ein Haus kauft, er wird einen anderen (B) darüber eine Pachturkunde ausstellen lassen, um für ihn es (juristisch) rein sein zu lassen. Siehe den Wortlaut der Pachturkunde: $(\mathbf{x}+11,24) \gg B$ sagte zu A: Ich (B) bin mit dir (A) für X Jahre, Y Monate, um zu wachen über dein Haus, welches in der und der Stadt ist, welches du gekauft hast $(\mathbf{x}+11,25)$ von C. Seine südlichen, nördlichen, östlichen, westlichen Nachbarn. ${ }^{11)}$ Und du gibst mir soundso viel Geld als mein Unterhalt. Zu meinen Lasten ist der Schaden deines Hauses.« Wenn man (x+11, 26) ihm (A) drei Pachturkunden ausstellt gemäß dem, was oben geschrieben ist, für drei Jahre - 36 Monate -, indem das Schriftstück, welches inm der Herr des Hauses (C) gemacht hat, in seiner Hand ist, ohne daß ein Mensch dagegen geklagt hat und ohne daß ein öffentlicher Protest gemacht wurde gegen ihn bezüglich dieses nämlichen Hauses, (x+1I, 27) ist es für ihn (juristisch) rein.

Wenn ein Mensch (A) einen Acker kauft, und man (B) ihm eine Pachturkunde ausstellt, um ihn (den Acker) ihm (A) (juristisch) rein sein zu lassen. 〈Siehe) den Wortlaut der Pachturkunde, die man ausstellt:

$(\mathbf{x + I I}, \mathbf{2 8 )} \gg B$ sagte zu A: >Du (A) hast mir (B) den und den Acker verpachtet, welcher in der und der Stadt ist, der für die und die Sache geeignet ist, welchen du von $C$ gekauft hast, (x+II, 29) dessen Süden, Norden, [Osten und Westen sind. 12) Und ich] bestelle sie in dem und dem Jahr, und du gibst [mir das Saatkorn] zu soundso viel Artaben für (eine Arure) Acker, und ich gebe dir die Ernteabgabe der obigen Äcker ( $\mathbf{x + 1 1 , 3 0 )}$ zu soundso viel Artaben für eine (Arure) Acker [...〈ca. $8 \mathrm{~cm}\rangle \ldots$, indem sie gemessen, $]$ ausgeliefert und gebracht werden zu dem und dem Ort. Wenn ich die obigen Felder

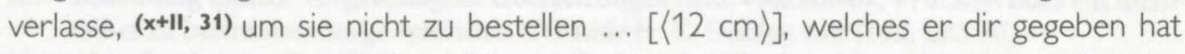
von obigem Saatkorn, und [ich (?)] werde dir den Rest geben, (x+II, 32) um [es (das Saatkorn?)] zu füllen. [lch] werde nicht [können ...〈ca. $12 \mathrm{~cm}\rangle \ldots]$ sie betreffend, indem 〈ich) nicht sagen kann »Erneuere 〈die Pacht) für ein Jahr«, bis du $(\mathbf{x}+$ III, $\mathbf{1})$ mir eine Quittung darüber ausgestellt hast.« Wenn man ihm drei Pachturkunden für drei Jahre -

11. In Urkunden werden hier die Namen der Nachbarn eingetragen.

12. In Urkunden stehen hier die Namen der Nachbarn. 
3[6 Monate - ausgestellt hat, während das Dokument, das ihm der Herr der Äcker (C) ausgestellt hat in seiner Hand ist, ohne daß irgendein Mensch dagegen geklagt hat und ohne daß man gegen ihn einen öffentlichen Protest erhoben hat bezüglich dieser nämli-

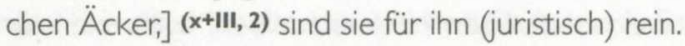

Es gibt einen Menschen, und man baut [ihm] H[äuser ...〈ca. $22 \mathrm{~cm}\rangle \ldots](\mathbf{x}+\mathbf{l l 1}, \mathbf{3})$ Ein Mensch kommt, und sie (die Häuser) werden ihm vermietet, und man gibt Geld pro [Monat als ihre Miete ... $\langle$ ca. $18 \mathrm{~cm}\rangle \ldots$

Siehe den Wortlaut der Pachturkunde, die man ausstellen wird:13)] $(\mathbf{x + 1 I I , 4 )} \gg \mathrm{A}$ [sagt] zu B: [>Du verpachtetest mir deine Weberei (?) ${ }^{14)}$, welche $\ldots\langle$ (ca. $13 \mathrm{~cm}\rangle \ldots$ für $X$ Jahre,] $(\mathbf{x + I I I , 5 )}$ Y Monate. Und ich gebe dir soundso viel Geld als ihre M[iete pro Monat ... <ca. $13 \mathrm{~cm}\rangle$... Ich werde nicht sagen können »lch habe dir Geld gegeben (oder) irgend etwas«,] $(\mathbf{x}+111,6)$ wenn du mir keine Quittung darüber ausgestellt hast. Ich werde nicht



$(\mathbf{x}+$ III, 7$)$ Wenn der Herr der Weberei15) klagt gegen den Menschen, [der die Pachturkunde für ihn ausgestellt hat über sie (die Weberei), sagend »Möge er mir die Gelder geben, über die er mir geschrieben hat«, wird man ihm (dem Pächter) sagen: »Veranschlage] $(\mathbf{x}+\mathbf{I I I}, \mathbf{8})$ die Stoffe als Geld.« Und man wird ihn schwören lassen über den

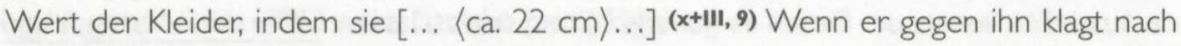
Ablauf [des Jahres der Pachturkunde .... und der,] gegen den [gekla]gt wird, [sagt: »lch habe nicht ...« $\langle$ ca. $5 \mathrm{~cm}\rangle \ldots$, lasse] $(\mathbf{x + l I I ,}, \mathbf{1 0})$ ihn mir schwören gemäß dem, was außerhalb (des Textes) geschrieben ist: >[lch] habe keinen Besitz über meinen und meiner Leute Bedarf hinaus bis zum letzten Tag des 3. Monats des Frühjahrs ${ }^{16)}$ [... (ca. $4 \mathrm{~cm}\rangle$... «] $(\mathbf{x + I I I , 1 1 )}$ Wenn man eine Pachturkunde über eine Brauerei ausstellt, handelt man gemäß dem Obigen. Man setzt nicht »Kleidung« ein, sondern er (der Pächter) soll sagen: »)/ch werde dir soundso viel Bier und [soundso viel] Mtk-Getränk geben [als ihre Miete pro Monat. Den Monat,] $(\mathbf{x + 1 I I , 1 2 )}$ in dem ich dir nicht Bier und Mtk-Getränk gebe, werde ich dir soundso viel Geld statt dessen geben.«

Wenn der Herr der Brauerei k[lagt gegen den Menschen, dem er die Pachturkunde ausgestellt hat,] $(\mathbf{x + I I , 1 3 )}$ sagend »Möge er mir mein Geld geben«, dann wird man zum Herren der Brauerei sagen: »Das Bier und die Mtk-Getränke, veranschlage sie als Geld.« Und man verfährt für ihn [ge]mäß der Pachturkunde über eine W[eberei ..., welche oben ist.] (x+III, 14) Wenn man über eine Gefügelzüchterei (?) eine Pachturkunde ausstellt, ist es gemäß dem, [was] oben geschrieben steht, wie man handeln soll.

Das Geld, über das er schreiben wird, ist das, welches man ihn ihm geben lassen wird. [Und er wird sagen:] »/ch [werde] (x+III, 15) dir soundso viele 10er Einheiten an Gänsen geben, und ich werde dir [soundso viele hn-Einheiten ${ }^{17)]}$ heißen Wassers geben als $[\ldots\langle$ ca. $5 \mathrm{~cm}\rangle \ldots]$ pro Tag.« Wenn [der Herr der] Geflügelzüchterei $(\mathbf{x}+\mathbf{I I I}, \mathbf{1 6})$ klagt gegen

13. Diese oder eine ähnliche Ergänzung dürfte nach ähnlichen Stellen sicher sein, ist aber nicht bei K. Donker van Heel (in: aaO 25) zu finden.

14. Unsicher, weil nur aus dem folgenden Zusammenhang erschlossen.

15. Zum Wort $m y_{3}$ s. P. W. Pestman, in: Enchoria 12 (1984) 40. So auch bei K. Donker van Heel, $\mathrm{aaO} 27$.

16. Aus dem spezifischen Datum mag auf eine Laufzeit von einem Jahr geschlossen werden (K. Donker van Heel, aaO 26).

17. Ein $h n$ ist ungefähr ein halber Liter. 
den Menschen, der ihm die Pachturkunde ausgestellt hat, sagend »Möge er mir das Geld für mein Haus geben«, wird man zum Herren des Hauses sagen: »Veranschlage die 10er Einheiten und [das] h[eiße] Wasser [als Geld.« Man] wird für ihn handeln gemäß dem, was außerhalb (dieses Textes) geschrieben ist. $(\mathbf{x}+$ III, 17) Wenn man eine Pachturkunde über einen Laden ausstellt, handelt man gemäß [der] obigen Pachturkunde.

Man setzt nicht »heißes Wasser« in sie (die Urkunde) hinein. Die Güter und das Geld, [worüber] er schreibt, [sind die, die man ihn ihm] geben läßt $(\mathbf{x + I I I , 1 8 )}$ gemäß der obigen Worte.

Wenn man eine Pachturkunde über ein šyš18) ausstellt, wird man entsprechend der obigen Pachturkunde handeln, indem man schreibt, um es (juristisch) rein sein zu lassen für den Herren des Hauses. Wenn er klagt [»Möge] er mir die Güter [für mein Haus geben,] (x+III, 19) worüber er mir geschrieben hat«, dann sagt man zu ihm: »Veranschlage es als Geld.« Man wird es inn gemäß dem geben lassen, was oben geschrieben ist.

Es gibt einen Garten, über [den] man einen Pachtvertrag macht, indem er [... 〈ca. $7 \mathrm{~cm}\rangle . .$. ] es geben seinem (des Gartens) (x+III, 20) Herrn gemäß dem obigen Vertrag, und er (der Pächter) soll sagen in der Pachturkunde, welche er ausstellt: »Und ich werde dir ein Bündel (?) Kraut ${ }^{19)}$ pro Tag geben. An dem Tag, an dem [ich] dir [nicht] geben [werde], werde [ich dir] soundso viel [Geld geben] an ( $\mathbf{x + I I I , 2 1 )}$ seiner Stelle.« Wenn der Herr des Gartens [klagt gegen den Menschen], der ihm die Pachturkunde ausgestellt hat, sagend »Möge er das Geld geben, über das er mir geschrieben hat [statt des] Bündels (?) Kraut $[\ldots \ll\langle$ ca. $8 \mathrm{~cm}\rangle \ldots],(\mathbf{x}+\mathbf{I I I}, \mathbf{2 2})$ dann wird man ihn es geben lassen gemäß dem, was oben geschrieben ist.

$[\ldots\langle$ ca. $4 \mathrm{~cm}\rangle$.... $]$ diese obigen Pachturkunden: Der Mensch, der von ihnen abgeht, bevor das Jahr [der Pachturkunde vollendet ist (?) .................. Wenn] (x+III, 23) der Herr des Hauses einen öffentlichen Protest gegen den Menschen macht, [der für ihn die Pachturkunde ausgestellt hat.20)]

Siehe den Wortlaut des öffentlichen Protests, der erhoben werden wird:21)

$(\mathbf{x}+\mathbf{I I}, \mathbf{2 4 )})$ Regierungsjahr $\mathrm{X}$, Monat $\mathrm{Y}, \mathrm{A}$ [erhebt einen öffentlichen Protest gegen B: 〉Er (B) hat mir (A) eine Pachturkunde über mein Haus ausgestellt, welches in der und der] Stadt ist, dessen Süden, Norden, [Osten und Westen sind,22) für] $(\mathbf{x}+\mathbf{I I I}, \mathbf{2 5}) \times$ Jahre, Y Monate bis zum [Monat] soundso [...〈ca. $4 \mathrm{~cm}\rangle \ldots$ Er hat in die] Pachturkunde [geschrieben,] die er mir gemacht hat: 》)/ch werde nicht [sagen können: >lch habe dir Geld gegeben] (x+III, 26) (oder) Güter dafür, indem du mir keine Quittung darüber ausgestellt hast.« [Er hat mir] nicht [Geld (oder) Güter als die Miete meines Hauses gegeben

18. Bedeutung unklar. Vorgeschlagene Übersetzungen sind: »Backofen«, »Vorhof« oder ein nicht näher zu bestimmendes gewerbliches Gebäude (s. K. Donker van Heel, aaO 30).

19. Als allgemeiner Begriff für ein unspezifisches demotisches Wort für »Pflanzen« $(s m)$. K. Donker van Heel (in: aaO 31) übersetzt »legumes«, was m. E. zu eng gefaßt ist.

20. Aufgrund des Platzbedarfs muß hier noch im Gegensatz zu K. Donker van Heel (in: aaO 33) ein Spatium angesetzt werden, wie auch in $\mathrm{x}+\mathrm{II}, 19$ in ähnlicher Situation.

21. Der bei K. Donker van Heel (in: aaO 33) nach dem Spatium gekennzeichnete Verlust an Papyrus war wohl nicht mit Text versehen, wie der Vergleich mit den anderen Einleitungsformeln und der Anfang des Beispieltextes in der folgenden Zeile zeigt. Das Spatium erstreckte sich also kurz nach der Kolumnenmitte beginnend bis zum Zeilenende.

22. In der Urkunde stehen hier die Namen der Nachbarn des Hauses. 
...〈ca. $10 \mathrm{~cm}\rangle$...] $(\mathbf{x}+$ III, 27) der Termin. [Er schuldet] mir soundso viel Geld (oder) soundso viele Güter [von] dem Geld, [über das] er [mir geschrieben hat in der Pachturkunde ...〈ca. $12 \mathrm{~cm}\rangle \ldots$... (x+III, 28) Möge er es mir geben. Ich fand ihn nicht, [um einen öffentlichen Pro]test in Anwesenheit gegen ihn zu erheben. Mein [öffentlicher Protest für jedes Jahr ist es.« Er soll 3 Jahre verbringen, indem er den öffentlichen Protest erhebt jedes Jahr für das Geld, weswegen 3 öffentliche Proteste erhoben werden nach der Art dessen, was oben geschrieben ist.]

(x+III, 29) Wenn der Mensch, der den öffentlichen Protest erhoben hat, klagt [gegen den Menschen, der] ihm die Pachturkunde [ausgestellt hat, sagend ...〈ca. $19 \mathrm{~cm}\rangle \ldots$ ] $(\mathbf{x + I I I}, \mathbf{3 0})$ Wenn ein Mensch klagt gegen einen Menschen, sagend $\gg E r$ hat mir eine Pachturkunde ausgestellt. Er ist fortgegangen, indem er ver[lassen hat mein Haus ... 〈ca. $17 \mathrm{~cm}\rangle \ldots . .].(\mathbf{x}+11, \mathbf{3 1})$ welche/r/s oben ist.

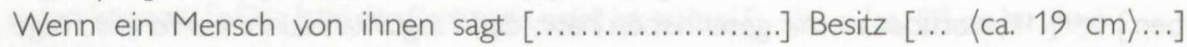
$(\mathbf{x + I I I , 3 2 )}$ sagend: $\gg / \mathrm{lch}$ habe ihm eine Quittung ausgestellt über das Geld (oder) die Güt [er ... 〈ca. $16 \mathrm{~cm}\rangle \ldots$

Siehe den Wortlaut der Quittung, die ausgestellt werden wird:]

$(\mathbf{x + 1 V}, \mathbf{1})[\gg \mathrm{A}$ sagt zu B:] >Du hast mir soundso viel Geld gegeben von soundso viel Geld, über das [du mir] eine Pachturkunde [ausgestellt hast], um es mir zu geben als das Geld meines Hauses, im Regierungsjahr $X$, Monat $Y(\mathbf{x + 1} \mathbf{v}, \mathbf{2})[\ldots \ldots \ldots \ldots \ldots \ldots \ldots$ und du mußt mir soundso viel Geld geben] als den Rest des Geldes, das du schuldest, über das du mir geschrieben hast als Geld meines [obigen] Hauses [im Regierungsjahr] X, Monat Y.« Wenn der Herr des Hauses sagt: 》lch habe nicht $(\mathbf{x + l v}, \mathbf{3})$ [ihm eine Quittung ausgestellt über den Rest des Geldes«, dann wird man sagen zu] dem Menschen: »Bringe die Quittung! Beweise seine Echtheit!23) « Wenn er [einen Echtheits]beweis [gibt] nach der Art des Echtheitsbeweises, welche angewendet wird, $(\mathbf{x}+\mathbf{I V}, \mathbf{4})$ [wird der Herr des] Hauses [veranlaßt werden, inm (dem Pächter)] das Geld gutzuschreiben, über welches er ihm geschrieben hat. Wenn er die Echtheit nicht beweist, akzeptiert man es nicht.

$(\mathbf{x + 1}, \mathbf{5})$ [Wenn der Beklagte sagt:] 》\Lasse mir den Herren des Hauses schwören über [den Rest des Geldes (oder) die] Güter, von denen er sagt >Du hast es mir nicht gegeben««, dann wird man ihn schwören lassen. Wenn er nicht $(\mathbf{x}+\mathbf{I V}, \mathbf{6})$ [schwört, dann wird man ihn das Geld (oder) die] Güter [empfangen lassen], über die er inm geschrieben hat.

Dotationsrecht.

Die Dotationsurkunden, die man ausstellt. Ihr Wortlaut:

$(\mathbf{x + I V}, \mathbf{7})$ [》Regierungsjahr $\mathrm{X}$, Monat $\mathrm{Y}$. A sagt] zu B: >Du (B) hast mir (A) [soundso viel Geld als Dota]tion meiner24) Frau C, Tochter des D, ihre Mutter ist E, gegeben, um dir soundso viel Geld zu geben $(\mathbf{x}+\mathbf{I V}, \mathbf{8})$ [als ihren Unterhalt pro Jahr in dem] Haus, welches du wünschst. Du bist der, der bevollmächtigt ist [über den Ausstand] ihres Unterhalts. ${ }^{25}$ )

23. Wörtlich: "Lasse sie auf dem Fuß stehen!«

24. K. Donker van Heel (in: aaO 38) zieht die Möglichkeit von $t_{5} s h m . t$ und $t_{5} y=k$ šr.t in Erwägung, beide Alternativen lassen sich mit dem Demotischen nicht vereinbaren. $t_{5} y=y$ shm.t würde allen Zeichen Rechnung tragen, so auch S. Grunert, Der Kodex Hermopolis und ausgewählte private Rechtsurkunden aus dem ptolemäischen Ägypten, Leipzig 1982, 58.

25. D. h. es wird B eingeräumt, vor Gericht einen etwaigen Ausstand einzuklagen. 
Alles, was ich habe, und das, was ich erwerben werde, sind das Pfand $(\mathbf{x}+\mathbf{I V}, \mathbf{9})$ [für ihren Unterhalt. Wenn man] von dir [einen Eid] verlangt, um ihn mir abzulegen an der Stelle, [an] der die R[ichter sind,] sollst du ihn mir ablegen.« «26)

Die Form dessen, wenn ein Mensch ihn ablegt und der Mensch, $(\mathbf{x + l v}, \mathbf{1 0})$ [dem er ihn abgelegt hat, sagt: »Er hat mir nicht den Aus]stand an Unterhalt gegeben.« Der Mensch, der die Urkunde ausgestellt hat, wird gefragt werden: »Diese Urkunde, [hast du sie ausgestellt?« Wenn er sagt:] »lch habe sie ausgestellt«, sagt man zu ihm: »Gib ihm den Unterhalt.« Wenn er sagt [»/ch habe] $(\mathbf{x}+\mathbf{I V}, \mathbf{1 1})$ nichts, [um für das Jahr zu geben, welches vorübergegangen ist«, dann wird] er durch sie (die Urkunde) [gezwungen werden,] um zu veranlassen, daß er ihm Unterhalt gibt für das nämliche Jahr. Und man wird [ihn Unterhalt geben] lassen [bis zu] dem Monat der Klage gegen ihn, welche man erhoben hat. Und man wird ihn Unter[halt] $(\mathbf{x}+\mathbf{I v}, \mathbf{1 2})$ geben lassen $[\ldots\langle\mathrm{ca} .5 \mathrm{~cm}\rangle$... Wenn] er klagt gegen ihn von Monat 3 des Sommers [des Jahres bis] Monat 4 des Sommers, wird man ihn ihm Unterhalt geben lassen bis zum Monat $(\mathbf{x + 1 v}, \mathbf{1 3})$ [der Klage gegen ihn, die erhoben wurde.]

Wenn der Herr der Urkunde sagt: »Es gibt viele Jahre, in denen [er mir nicht] Unterhalt gegeben hat《, und wenn der Beklagte sagt $(\mathbf{x}+\mathbf{I v}, \mathbf{1 4})[\gg \ldots\langle$ ca. $4 \mathrm{~cm}\rangle \ldots]$ Ich schulde ihm [keinen] Unterhalt«, dann setzt man drei Jahre an gemäß der Aussage (?) des Herren der Urkunde, [und ebenso in dem Jahr,] in [dem] er geklagt hat, um 4 Jahre voll zu machen. Und man läßt $(\mathbf{x + l v}, \mathbf{1 5})$ [ihn dem Menschen schwören, der die Urkunde ausgestellt hat:] »Du hast mir keinen Unterhalt gegeben für die Jahre.« Wenn er schwört, wird man zu dem Menschen sagen, der [die Urkunde ausgestellt hat: »Gib ihm Unterhalt] für die drei Jahre, über die er dir geschworen hat. « Wenn er sagt (x+IV, 16) [》》lch habe nichts, um ihm zu geben«,] dann wird man ihn schwören lassen: »lch habe keinen Besitz über meinen und meiner Leute Bedarf hinaus [bis zum Monat drei des Frühjahrs, letzter

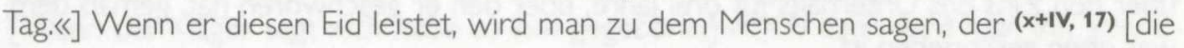
Dotationsurkunde ausgestellt hat:] »Schreibe über die Jahre, welche über die drei Jahre hinausgehen, von dem er sagt: >Mir wurde nicht in [ihnen] Unterhalt gegeben.« [Wenn er sagt 》)lch gab ihm Unterhalt für] die Jahre, welche über die drei obigen Jahre (x+IV, 18) hinausgehen«, [wird man ihn schwören lassen:] »Du hast [keine] Schuldigkeit gegen mich in ihnen.« Wenn er diesen Eid leistet, wird man ihn (über) den Unterhalt [für die] 3 [Jahre] schreiben lassen, welche oben geschrieben sind. Wenn er keinen Eid leistet über den Unter-(x+IV, 19) [halt für die Jahre, welche hinausgehen über] die [drei] Jahre,

26. Zur Verdeutlichung der Konstellation dieses wichtigen Kapitels des Kodex: Der Mann (B) eines Paares (B und C) trifft eine Vereinbarung mit einem anderen Mann (A), der für die Frau (C) handelt. A zahlt dem B einen Betrag (Dotation), wodurch B verpflichtet ist, für den Unterhalt der Frau C aufzukommen. Die tatsächliche Rechtspraxis im ptolemäischen Ägypten, wie sie sich in den Urkunden spiegelt, zeigt aber, daß vielmehr die Frauen ohne Vermittler die Vereinbarungen mit ihrem Partner direkt trafen. Das einzige erhaltene Dokument, das dem Modell des Hermupolis-Kodex folgt, ist der Papyrus Bibliothèque nationale 219a (316 v. Chr.), dort ist A der Vater von C, doch gibt es Hinweise darauf, daß diese Praxis in der 26. Dynastie häufiger Anwendung fand, sie aber in ptolemäischer Zeit nicht mehr in Gebrauch war. ( $\mathrm{Zu}$ den Dotationsurkunden $(s h n s i n h)$ s. zuletzt mit weiterer Literatur: J. H. Johnson, »Annuity Contracts« and Marriage, in: D. P. Silverman (ed.), For His Ka. Essays in Memory of Klaus Baer, SAOC 55, Chicago 1994, 113-132). 
wird man es hinzusetzen zu dem Unterhalt der obigen drei Jahre, indem man ihn schreiben läßt entsprechend dem, was [oben] geschrieben ist.

[Siehe den Wortlaut der Urkunde, die man ausstellen wird über] den Ausstand des Unterhalts:

$(\mathbf{x + I V , 2 0 )}$ [》》Regierungsjahr X, Monat Y. A sagt zu] B: >lch (A) schulde dir (B) soundso viel Geld als Geld an Ausstand für den Unterhalt [für drei Jahre ...〈ca. $4 \mathrm{~cm}\rangle \ldots$, welche] ich [dir nicht] $(\mathbf{x + 1 v}, \mathbf{2 1})$ gegeben habe $[\ldots\langle\mathrm{ca} .4 \mathrm{~cm}\rangle \ldots$ Ich werde] es dir geben 〈gemäß dem), was in der Dotationsurkunde geschrieben ist, die ich dir ausgestellt habe mit dem und dem Zins im Regierungsjahr soundso, [Monat soundso ...〈ca. $4 \mathrm{~cm}\rangle$... Dotat]ion $(\mathbf{x}+\mathbf{I V}, \mathbf{2 2})[\ldots\langle$ (ca. $4 \mathrm{~cm}\rangle \ldots$ Und ich werde] dir das Geld [geben] und die Kleider, die ich dir nicht gegeben habe und die oben sind für $X$ Jahre, $Y$ Monat $[\ldots$ <ca. $4 \mathrm{~cm}\rangle \ldots]$, was oben ist $(\mathbf{x + l v}, \mathbf{2 3})[\ldots\langle\mathrm{ca} .4 \mathrm{~cm}\rangle \ldots . \times]$ Jahre von Monat soundso an, 〈indem) das Geld Zins trägt gegen mich zur Rate von 11/2 zu 1 zum Monat 3 des Frühjahrs pro Jahr. $\left.{ }^{27}\right) \ldots[\ldots\langle\text { ca. } 4 \mathrm{~cm}\rangle \ldots]^{28)}(\mathbf{x}+\mathbf{I V}, 24)[\ldots\langle\mathrm{ca} .4 \mathrm{~cm}\rangle \ldots$ Und $]$ ich werde es dir geben (als) Zins gegen mich. Wenn ich dir das obige Geld und seine obigen Zinsen nicht gebe, sind sie gegen [mich und meine Kinder ge]mäß dem Papyrus, $(\mathbf{x + 1}, \mathbf{2 5})$ [welchen ich dir ausgestellt habe.«

Wenn der] Mensch, [der] die Urkunde ausgestellt hat, [zu dem Menschen,] für den er die Urkunde ausgestellt hat, sagt: »Du bist nicht zu mir gekommen wegen deines Unterhalts für die Jahre, [wegen] der du ge[gen mich] klagst [ .....................

$(\mathbf{x}+\mathbf{I} \mathbf{v}, \mathbf{2 6})$ [Wenn der, der] bevollmächtigt ist [über den Ausstand,] schwört über den Unterhalt für drei Jahre, und er einen Eid darüber leistet wegen Zins »lch bin zu dir ge-

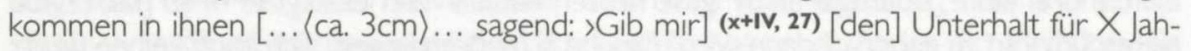
re. Du hast es mir nicht [gegeben] , dann wird man ihn eine Urkunde ausstellen lassen über den Zins des Unterhalts für drei Jahre, über den er geschworen hat, gemäß dem, was oben geschrieben ist.

[Wenn der Mensch, der die Urkunde ausgestellt hat,] einen $(\mathbf{x + I V}, \mathbf{2 8})$ [Ei]d leistet für den Herren der Urkunde wegen der Jahre über die drei Jahre hinaus $\gg D u$ bist nicht zu mir gekommen wegen des dir in ihnen (sc. den Jahren) zustehenden Unterhalts«, und sie [den Menschen befragen ... 〈ca. $4 \mathrm{~cm}\rangle \ldots$...]

$(\mathbf{x + I v}$, 29) Wenn der Beklagte sagt »lch habe inn voll bezahlt mit dem Unterhalt. Er hat mir eine Quittung darüber ausgestellt«, dann wird man zu ihm sagen: »Bringe die Quit-


über.« Wenn seine Quittung echt ist,

siehe den Wortlaut der Quittung, die man machen wird: »A sagt [zu B: >Du hast mir gegeben] (x+lv, 31) [...(ca. $4 \mathrm{~cm}\rangle \ldots$ Geld] und soundso viel Zins im Regierungsjahr $X$, Monat $\langle Y\rangle$, indem ich noch Anspruch gegen dich habe (auf) die Dotation.« W[enn der Mensch, gegen den] $(\mathbf{x + 1} \mathbf{v}, \mathbf{3 2})$ [geklagt wird,] sagt »Er hat mir eine Quittung darüber ausgestellt«, dann wird man den Herren der Urkunde befragen. Wenn er sagt »Es wurde

27. Gegen K. Donker van Heel (in: aaO 45), der hier $t n 11 / 2 r 1 r m s$ hr rnp.t»(...) at the rate of $50 \%$ shall bear interest annually liest, steht hier $\operatorname{tn} 1 \frac{1 / 2}{r} 1 \mathrm{r} 3 \mathrm{bd} 3$ h.t hr rnp.t.

28. Der Verlust ist hier etwas unsicherer, weil offenbar noch ein Spatium am Zeilenende gelassen wurde. 
mir nicht gegeben«, dann wird man ihn einen Eid leisten lassen: »Du hast [es] nicht gegeben.《

[Siehe den Wortlaut des Eides, welchen man verlangt] $(\mathbf{x}+\mathbf{v}, \mathbf{1})$ von einem Menschen, welcher vor den Richtern ist, [die den] Eid verlangen von [ihm]: »Du hast [es nicht gegeben.«] Die Richter sagen dann zu [dem Menschen, der die Urkunde ausgestellt hat:] $(\mathbf{x}+\mathbf{v}, \mathbf{2}) \gg \operatorname{Der}[$ nämliche] Unterhalt, [gib ihn] dem Herren [der Urkunde.《] Man zwingt [ihn], bis man einen Erlaß geschrieben hat auf seine (des Klägers) Aussage hin: »Du hast es mir gegeben.《

$(\mathbf{x}+\mathbf{v}, \mathbf{3})$ Wenn ein Mensch klagt gegen einen Menschen: »Er hat mir über eine Dotation über soundso viel Geld 〈an〉 Unterhalt geschrieben. Er hat es mir nicht gegeben.« Wenn der Beklagte [sagt »/ch habe ihm nicht über eine Dotation geschrieben«, dann sagt man] $(\mathbf{x}+\mathbf{v}, \mathbf{4})$ zum Kläger: »Beweise die Echtheit deiner Urkunde.« Wenn er die Echtheit beweist, wird man zu dem Beklagten sagen: »Gib ihm Unterhalt [gemäß der Urkunde, um es] ihm $(\mathbf{x}+\mathbf{v}, \mathbf{5})$ [zu geben,] welche du ausgestellt hast. « Wenn er sagt »lch habe nichts, um zu geben für das vergangene Jahr, aber ich gebe ihm Unterhalt für das Jahr, in dem er gegen mich geklagt hat«,

[wird man ihn schwören lassen: $(\mathbf{x}+\mathbf{v}, \mathbf{6})$ ) $\gg$ /ch habe keinen Besitz über meinen und meiner Leute Bedarf hinaus bis zum [Monat 3] des Frühjahrs, letzter Tag.« Man läßt ihn darüber schreiben, indem es Zinsen trägt gemäß dem, was geschrieben ist zu Zinsgeld, welches [oben] geschrieben steht. [Man wird veranlassen,] $(\mathbf{x}+\mathbf{v}, \mathbf{7})$ daß er Unterhalt gibt für das Jahr, in dem er gegen ihn geklagt hat.

Wenn ein Mensch klagt gegen einen Menschen: »Er hat mir geschrieben über eine Dotation, indem er [mir] nicht [Unterhalt] gegeben hat.«29) [Wenn der] $(\mathbf{x}+\mathbf{v}, \mathbf{8})[\mathrm{Be}]$ klagte sagt »Die Urkunde, die ich ihm ausgestellt habe, er hat mir nicht das Geld gegeben, [über] das ich ihm geschrieben habe [ ..〈ca. $3 \mathrm{~cm}\rangle \ldots \ll$,$] dann wird der Kläger befragt.$ [Wenn der Kläger sagt »»ch habe es ihm gegeben«,] $(x+\mathbf{v}, 9)$ dann wird man ihn schwören lassen: „Soundso viel Geld, welches in der Urkunde geschrieben steht, die du mir gemacht hast, und von dem du sagst: > [Er] hat es mir nicht [gegeben.< Ich habe] es dir [gegeben], ich habe [dich damit] voll bezahlt. Ich schulde $(\mathbf{x}+\mathbf{v}, \mathbf{1 0})$ [dir nichts.] Wenn er diesen Eid ablegt, wird man den Beklagten die [Bestimmung der Urkunde, die er] ihm [ausgestellt hat,] ausführen lassen gemäß dem, was oben geschrieben ist. [Wenn er diesen Eid nicht ablegt,] $(\mathbf{x}+\mathbf{v}, \mathbf{1 1})$ wird man die Urkunde vor dem Beklagten zerreißen.

Wenn der Beklagte sagt »Die Urkunde, die ich [für ihn ausgestellt habe, er hat mir nicht gegeben] $(\mathbf{x}+\mathbf{v}, \mathbf{1 2})$ das Geld, welches geschrieben ist in nämlicher Urkunde. Soundso viel Geld ist zu seinen Lasten noch übrig. Er hat es [mir] nicht gegeben«, dann wird man [den Herren] der Urkunde fragen. Wenn er sagt: »Soundso viel Geld, [ich habe es inm gegeben.] Ich schulde $(\mathbf{x}+\mathbf{v}, \mathbf{1 3})[\mathrm{ihm}]$ soundso viel Geld durch die Dotationsurkunde«, dann läßt man den Menschen, der [die Urkunde ausgestellt hat, Unterhalt] geben gemäß dem Geld, das inm gegeben wurde.

$(\mathbf{x}+\mathbf{v}, \mathbf{1 4})$ Wenn der Mensch, der die Urkunde ausgestellt hat, klagt gegen den, für den er

29. Die Ergänzung von $r b n-p w=f t^{3}\left[-n=y^{\circ} q-h b s(\ldots)\right]$ weicht von der K. Donker van Heels (in: $\mathrm{aaO} 53) \mathrm{ab}$, der nur $r$ bn-pw=f ti $[-s(\ldots)]$ ergänzt und an der Stelle $(\ldots)$ without having given [it (...)] « übersetzt. Ein einfacher pronominaler Rückbezug, der auf »Dotation« zielte, reicht hier aber nicht aus, denn die Dotation ist ja gezahlt worden, aber der daraus abgeleiteten Unterhaltspflicht ist nicht Genüge getan worden. 
die Urkunde ausgestellt hat: »lch habe ihm über eine Dotation [geschrieben. Das Geld,

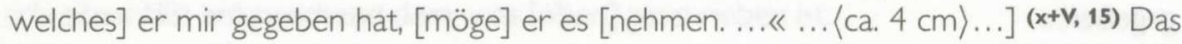
Geld, welches ihm gegeben wurde, man läßt ihn es dem geben, dem er die Urkunde ausgestellt hat, zusammen mit dem Unterhalt, welcher ihm zusteht bis zu dem Monat, [in] dem er geklagt hat.

[Wenn ein Mensch] $(\mathbf{x}+\mathbf{v}, \mathbf{1 6})$ einem Menschen bezüglich eines Ausstandes vom Unterhalt in einer Dotationsurkunde schreibt, weil er es nicht geben konnte, dann handelt man gemäß der Urkunde, bis er den Ausstand [des Unterhalts] gegeben hat.

$(\mathbf{x}+\mathbf{v}, \mathbf{1 7})$ Wenn ein Mensch klagt gegen einen Menschen über einen Ausstand [...] Unterhalt $[\ldots\langle$ ca. $3 \mathrm{~cm}\rangle \ldots]$, richtet [man] ihn nicht, wenn er nicht [die] Dotationsurkunde gefunden hat $[\ldots\langle\mathrm{ca} .4 \mathrm{~cm}\rangle \ldots](\mathbf{x}+\mathbf{v}, \mathbf{1 8})[$ Wenn] ein Mensch klagt gegen einen Menschen 》) Möge er mir das Geld geben, das ich ihm gegeben habe, so daß ich die Urkunde gebe, die er mir ausgestellt hat«, und der Beklagte [sagt »lch habe nichts, um ihm zu geben«,] $(\mathbf{x}+\mathbf{v}, \mathbf{1 9})$ dann wird man ihn einen Eid ablegen [lassen:] »/ch habe kein Geld über meinen und meiner Leute Bedarf hinaus bis [zum Monat 3 des Frühjahrs, letzter Tag.«] Man wird ihn schreiben lassen [über den Ausstand des Unterhalts,] $(\mathbf{x}+\mathbf{v}, \mathbf{2 0})$ und die Dotationsurkunde soll in der Hand des Klägers bleiben, indem man [den, der die Urkunde ausgestellt hat, das Geld] nicht (zurück)geben läßt. [ ..〈ca. $5 \mathrm{~cm}\rangle \ldots]$

$(\mathbf{x}+\mathbf{v}, \mathbf{2 1})$ Wenn der Mensch, für den die Dotationsurkunde ausgestellt wurde, gestorben ist, ohne daß er [für] einen anderen geschrieben hat bezüglich [der Dotations] urkunde im Namen der Frau, für die die Urkunde ausgestellt wurde $[\ldots\langle\mathrm{ca} .2 \mathrm{~cm}\rangle \ldots]$ $(\mathbf{x}+\mathbf{v}, \mathbf{2 2})$ Wenn die Frau klagt gegen den Menschen, der die Urkunde ausgestellt hat, »Möge er mir Unter[halt] geben [für das] vergangene [Jahr]«, [dann wird man ihn ihr geben lassen] $(\mathbf{x}+\mathbf{v}, \mathbf{2 3})$ den Ausstand an Unterhalt.

Wenn die Frau sagt »Lasse inn [mir Unterhalt] geben [für das vergangene Jahr«, und wenn der Beklagte] $(\mathbf{x}+\mathbf{v}, \mathbf{2 4})$ sagt »lch habe nichts«, dann wird man ihn schwören lassen gemäß dem, was oben geschrieben ist. Man wird ihn eine Urkunde schreiben lassen über das Geld. Man wird anordnen [das, was] oben geschrieben ist.

[Wenn ein Mensch klagt gegen einen Menschen] $(\mathbf{x + v}, \mathbf{2 5}) \gg$ Er hat mir über eine Dotation geschrieben, möge er mir Unterhalt geben«, und wenn der Beklagte sagt »Die Frau, für die die Urkunde ausgestellt wurde, sie ist to[t«, dann wird man den Kläger fragen,] $(\mathbf{x}+\mathbf{v}, \mathbf{2 6})$ und wenn er sagt: »Es ist die Wahrheit, die Frau ist tot.« Man läßt ihn ihr nicht Unterhalt geben. Man wird zu ihm sagen: »Das Geld, das er dir zuvor gegeben hat, gib es ihm (zurück) ......... [Wenn er sagt »lch habe nichts«, dann wird man ihn einen Ei]d $(\mathbf{x}+\mathbf{v}, \mathbf{2 7})$ [ablegen lassen.] Man wird ihn darüber schreiben lassen. Man wird [handeln ge]mäß dem, was oben geschrieben ist. Die, die sie für ihn schreiben, werden die Dotationsurkunde in seiner Hand lassen, bis er [es erfüllt. ............ ]

$(\mathbf{x}+\mathbf{v}, \mathbf{2 8})$ [Wenn] der Herr der Dotationsurkunde sagt $\gg$ Die Frau, von der er sagt, sie sei tot, sie lebt«, dann wird man zu ihm sagen: »Bringe die Frau vor die [Richter ......... Wenn er] einen Ei [d ablegt,] $(\mathbf{x}+\mathbf{v}, \mathbf{2 9})$ wird er (?) $[\ldots\langle$ ca. $3 \mathrm{~cm}\rangle \ldots]$ sagen $[\gg \ldots\langle$ ca. $4 \mathrm{~cm}\rangle . .$.$] welches in der Dotationsurkunde ist, die [du] mir mit ihr gemacht hast im$ Regierungsjahr $[X$, Monat $Y \ldots\langle\mathrm{ca} .3 \mathrm{~cm}\rangle \ldots \ll]$

$(\mathbf{x + v}, \mathbf{3 0})$ [Wenn] der Mensch, der die Do[tations]urkunde ausgestellt hat, gestorben ist, und [der, für den er die] Urkunde [ausgestellt hat,] klagt 〈gegen〉 seinen Sohn: »Dein Vater hat mir über eine Dotation geschrieben.« $(x+v, 31)$ [Wenn er sagt »Die] Urkunde, 
mein Vater hat sie gemacht«, dann wird man zu ihm sagen: »Gib ihm das Geld, welches geschrieben ist in der Urkunde. Gib ihm den Besitz deines Vaters.« [ .. 〈ca. $4 \mathrm{~cm}\rangle \ldots]$ $\left.(\mathbf{x}+\mathbf{V I}, \mathbf{1}){ }^{30}\right)[\ldots \ldots \ldots . . . k]$ lagen gegen ihn, ist, was getan werden wird. Wenn sie sagen: »Wir werden nicht prozessieren als die zwei Parteien - die Menschen, die für uns geschrieben haben bezüglich des Hauses, sind die, die prozessieren werden $(\mathbf{x}+\mathbf{V I}, \mathbf{2})[\mathrm{be}-$ züglich des nämlichen Hauses als die zwei Parteien«], werden die Rich[ter] zu ihnen sagen: »Klagt gegen sie.« Die zwei Parteien, die für sie geschrieben haben bezüglich des Hauses, werden gebracht werden. Sie sind die, die prozessieren als die zwei Parteien. $(\mathbf{x}+\mathbf{v} \mathbf{l}, \mathbf{3})$ [Sie werden von ihnen, nämlich beiden Parteien, einen Eid verlangen.] Sie werden den, der gelogen hat, eine Abstandsschrift für den, der wahr gesprochen hat, ausstellen lassen.

Wenn ein Mensch (A) klagt gegen einen Menschen (B):31) »B hat gebaut ( $\mathbf{x}+\mathbf{V I}, \mathbf{4})$ [auf einem Bauplatz für ein Haus, aber] der nämliche Bauplatz, er gehört mir (A), (weil) er meinem Vater gehörte. Er hat mir darüber geschrieben.« Wenn der Beklagte sagt »Er gehört mir, der Bauplatz $(\mathbf{x}+\mathbf{V} \mathbf{l}, \mathbf{5})$ [meines Vaters ist es. Er hat mit darüber geschrieben«, dann werden die Rich]ter zum Beklagten sagen: »Kannst du den Beweis erbringen, daß der Bauplatz dir gehört, weil er deinem Vater gehörte, $(\mathbf{x}+\mathbf{V I}, \mathbf{6})$ [und daß man dir darüber geschrieben hat? « Oder man wird] den Kläger [veranlassen], den Beweis zu erbringen, daß er ihm gehört, (weil) er seinem Vater gehörte und ihm darüber geschrieben wurde. Das, was der Beklagte wünscht, $(\mathbf{x}+\mathbf{v} \mathbf{l}, \mathbf{7})$ [ist das, was man] macht. Wenn er (der Beklagte) sagt »lch werde den Beweis bringen«, und wenn er den Beweis nicht bringt, wird man das Haus dem Kläger ${ }^{32}$ ) geben. Er (der Beklagte) wird [[nicht]] ${ }^{33)}$ ihm $(\mathbf{x}+\mathbf{v l}, \mathbf{8})$ [eine Abstandsschrift] schreiben.

Wenn der Beklagte zu den Richtern sagt »Lasse den Kläger diesen Beweis bringen, daß dieses Haus sein $(\mathbf{x}+\mathbf{v} \mathbf{l}, \mathbf{9})$ [Haus ist. ................, dann] werden [die Rich]ter zum Kläger sagen: »Wenn der Nutzen bei dir ist, bringe einen Beweis, daß das Haus dir gehört. «Wenn er einen Beweis bringt, dann wird man [ihm] $(\mathbf{x}+\mathbf{V I}, \mathbf{1 0})$ [das Haus geben und seinen] Prozeßgegner [ihm eine Abstandsschrift] schreiben lassen.

Wenn der Mensch sagt »Möge man mich meine Baumaterialien dieses Hauses hinausbringen lassen«, dann wird man ihn sie hinausbringen lassen. ${ }^{34)}$ Man wird $(\mathbf{x}+\mathbf{V I}, \mathbf{1 1})[\ldots$ 〈ca. $2 \mathrm{~cm}$ 〉...] ihn.

Wenn der Kläger keinen Beweis bringt für das Haus, wird man das Haus dem geben, der es gebaut hat. Man wird ihn [ihm] eine Abstandsschrift schreiben lassen. $(\mathbf{x}+\mathbf{V} \mathbf{l}, \mathbf{1 2})$ [Wenn ein Mensch ( $A$ ) gegen einen Menschen (B) klagt bezüglich] eines Hauses und er sagt: »Das Haus, sein Herr (C) ist der, der es mir (A) gegeben hat (als) Pfand für Getreide, welches er mir schuldet. $\operatorname{Er}(C)$ hat mir darüber geschrieben. [Er hat es mir nicht gegeben.] $(\mathbf{x}+\mathbf{V I}, \mathbf{1 3})$ [Das Haus ist mein Haus wegen] des Gebens als Pfand in meine Hand.

30. Zwischen den Kolumnen $x+V$ und $x+V I$ fehlt eventuell eine weitere Kolumne, so daß Kolumne $x+V I$ eigentlich $x+V I I$ ist. Ab hier beschäftigt sich der Text mit Baurecht.

31. Ab diesem Satz bis $x+V I, 11$ liegt eine griechische Parallele (Papyrus Oxyrhynchos XIVI.3285) vor. S. dazu: P. W. Pestman, Textes et études de papyrologie grecque, démotique et copte. P. L. Bat. 23, 116-143.

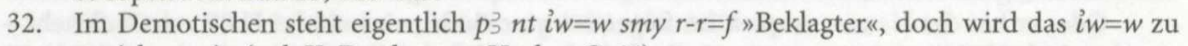
streichen sein (vgl. K. Donker van Heel, aaO 66).

33. Ein vom Schreiber selber bemerkter Fehler, den er ausbesserte.

34. Ende des Bereichs, für den die griechische Parallele vorliegt. 
Das Geld und das Getreide, welches er mir schuldet, er hat es mir nicht gegeben«,

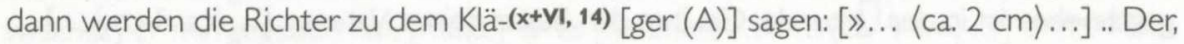
gegen den du klagst (B): > Er hat mein Haus genommen.< Bringe ihn zu uns.《

Wenn das Haus nicht für ihn (juristisch) rein ist, werden die R[ichter] (x+VI, 15) [zu dem Kläger sagen: »Du (A) wirst] klagen gegen den Menschen (C), der dir eine Urkunde über das Haus ausgestellt hat.« Wenn der Mensch klagt gegen den Herren [des] Hauses $(\mathbf{x}+\mathbf{V l}, \mathbf{1 6})[\ldots\langle c \mathrm{ca} .8 \mathrm{~cm}\rangle \ldots] \ldots \ldots \ldots . .$. vor den Richtern: $\gg D$ Das Haus, sein Herr hat es mir als Pfand für Geld (und) Getreide gegeben. $(x+v l, 17)[\ldots\langle c a .6 \mathrm{~cm}\rangle \ldots$ Die Richter zwingen] den Herren des Hauses, [bis er] den Kläger zufrieden[stellt.] We $[\mathrm{nn}](\mathbf{x}+\mathbf{V I}, \mathbf{1 8})[\ldots\langle\mathrm{ca} .8 \mathrm{~cm}\rangle \ldots]$ Er hat gegeben $[\ldots\langle\mathrm{ca} .7 \mathrm{~cm}\rangle \ldots]$ Wenn der Mensch, dem man das Haus gegeben hat [...]

[... 〈Zeilen 19-31 nur bis auf wenige Reste, die keine Rekonstruktion erlauben, erhalten oder völlig verloren $\rangle. .$.

$(\mathbf{x}+\mathbf{V} \mathbf{I I}, \mathbf{1})$ befragen den [Käufer. Wenn er sagt: »Das Gel]d, ich schulde es ihm 〈als $\rangle$ Rest.《 Wenn der Herr des Hauses, [der] das Haus verkauft [hat], sagt »Möge er das Geld zurücknehmen, das er mir gegeben hat $(\mathbf{x}+\mathbf{v i l}, \mathbf{2})$ als [Preis meines Hauses (?) ... (ca. $4 \mathrm{~cm}\rangle$... «], dann werden die Richter ihn das Geld geben lassen, das ihm gegeben wurde als Preis des Hauses, dem, der es ihm gegeben hat. Man wird seinen Prozeßgegner fern sein 〈lassen〉 $(\mathbf{x}+\mathbf{V} \mathbf{I I}, \mathbf{3})$ von dem [Haus.] Wenn der Herr des Hauses sagt »Möge er mir das Geld geben, welches der Rest des Preises meines Hauses ist«, dann läßt man ihn es ihm geben. Das, was dem Herren des Hauses nützt, tut er. $(\mathbf{x}+\mathbf{V I I}, \mathbf{4})$ Wenn der Beklagte, der der Herr des Hauses wurde, sagt »lch schulde ihm kein Geld«, dann wird man ihn schwören lassen:

»lch schulde inm kein Geld.«

$(\mathbf{x}+\mathbf{V I I}, \mathbf{5})$ Wenn das [Haus drei Jahre im Besitz des Menschen ist, der es gekauft hat,] ohne daß man gegen ihn geklagt hat, und man (dann) gegen ihn klagt, das Geld, von dem er sagt

»lch schulde es ihm«,

ist das, was man ihm geben wird. Wenn er sagt »Kein Geld $(\mathbf{x}+\mathbf{V I I}, \mathbf{6})$ ist ausgeblieben [für] ihn [als Rest zu meinen Lasten«, dann läßt man ihn schwören:] »Kein Geld ist ausgeblieben für ihn zu meinen Lasten.«

Wenn ein Mensch klagt gegen einen Menschen »Er hat mein Haus genommen. Es ist meines, $(\mathbf{x}+\mathbf{V I I}, \mathbf{7})$ es gehörte meinem Vater, [er hat mir darüber geschrieben«, und wenn der Bekl]agte sagt »Es ist meines. Es vom Fluß heraufbringen, ist das, was ich gemacht habe. $\left.{ }^{35}\right)$ Wenn es sich bis zum Fluß erstreckt, wird man zu dem Beklagten sagen: $(\mathbf{x}+\mathbf{V I I}, \mathbf{8})$ [》/Kannst du einen Beweis bringen, daß das Haus] deines ist und daß du es heraufgebracht hast vom Fluß? Oder man wird den dich Anklagenden den Beweis bringen

35. Der Satz ist in seiner Bedeutung nicht ganz klar. Entweder ist gemeint, daß der, der Anspruch auf das Haus erhebt, die Nilschlammziegel vom Fluß her geholt hat, um das Haus zu bauen. Oder er hat das Haus - es spielt im folgenden eine Rolle, wie nah das Haus am Fluß steht nach der Nilüberschwemmung wieder freigelegt und dadurch einen Anspruch auf das Haus erworben. D. h. der, der sich nicht um die Immobilie gekümmert hat, kann seinen Anspruch verlieren. 
lassen, daß es seines ist (und daß es) seinem ( $(\mathbf{x}+\mathbf{V I I}, \mathbf{9})$ Vater gehörte, [daß ihm darüber geschrieben wurde.« ...〈ca. $9 \mathrm{~cm}\rangle$... We]nn sich der Fluß nicht bis zum Haus erstreckt, dann wird man zu dem Kläger sagen: »Bringe einen $\mathrm{Be}-(\mathbf{x}+\mathbf{V I I}, \mathbf{1 0})$ [weis, daß es deines ist (und daß) es deinem Vater gehörte, daß er dir darüber geschrieben hat.« Wenn er einen Beweis bringt über das] Haus, wird man veranlassen, daß der Beklagte eine Abstandsschrift ausstellt bezüglich des nämlichen Hauses. (x+VII, 11) [Wenn der Klä]ger [keinen Beweis bringt,] dann wird man [ihn ihm eine Abstandsschrift über das nämliche Haus] ausstellen lassen.

Wenn der Kläger sagt »Das Haus, es ist meines, mir hat $(\mathbf{x}+\mathbf{V I I}, \mathbf{1 2})$ [mein Vater darüber] geschrieben《, [und wenn der Beklagte sagt »Das] Haus, es ist meines, man hat mir darüber geschrieben«, dann wird man zu dem Beklagten sagen: $(\mathbf{x}+\mathbf{V I I}, \mathbf{1 3})$ »Kannst du einen Beweis bringen, [daß das Haus deines ist? Sonst wird man den] dich [Anklagenden] einen Beweis bringen lassen.« Das, was ihm nutzt, man wird [es] entscheiden

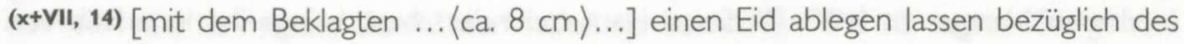
Klägers: »Das Haus ist meines. [Man hat mir] darüber [geschrieben], der Soundso, Sohn des Soundso, hat es widerrechtlich genommen. « Wenn ( $\mathbf{x + V I I , 1 5 )}$ [der] Beklagte sagt »Das Haus ist mein Haus«, 〈wohingegen) der Mensch, der sagt »Er hat mir darüber geschrieben«, [sagt »lch habe ihm nicht darüber geschrieben«,] (und) wenn kein Mensch von ihnen antwortet auf seine Worte: »Das Haus, es kam (x+VII, 16) [in meinen Besitz. ...〈ca. $7 \mathrm{~cm}\rangle$...] auf unsere Aussagen der zwei Männer«, dann wird man [auf sie $^{36)}$ sehen. Der, für den geschrieben worden ist, man wird inm das Haus geben] und man wird seinen Prozeßgegner eine Abstandsschrift schreiben lassen bezüglich des [nämlichen] Hauses. $(\mathbf{x}+\mathbf{V I I}, \mathbf{1 7})[\ldots\langle$ ca. $16 \mathrm{~cm}\rangle \ldots]$ in bezug auf das Haus, dann wird man ihn zwingen, bis er sein Herz zufriedenstellt. $(\mathbf{x}+\mathbf{V I I}, \mathbf{1 8})$ Wenn ein Mensch [einen Menschen] behindert [durch Errichten seines Hauses, sagend »... 〈ca. $13 \mathrm{~cm}\rangle . .$. ] Mein Haus ist es«, oder: »lch habe 〈einen Anspruch〉 darauf«, wohingegen der, der gebaut hat, $(\mathbf{x}+\mathrm{VII}, \mathbf{1 9})[\ldots\langle\mathrm{ca} .13 \mathrm{~cm}\rangle \ldots$

Wenn der Mensch, den man be]hindert, sagt »Er behindert durch Errichten des Hauses, $(\mathbf{x}+\mathbf{V I I}, \mathbf{2 0})$ obwohl er keinen Anspruch auf mein Haus hat. Soundso viel Geld ist mir verlorengegangen durch dieses Werk, an dem er mich behindert«, dann wird man ihn richten zusammen mit dem, der behindert hat $(\mathbf{x}+\mathbf{V I I}, \mathbf{2 1})$ sein Haus, gemäß dem, was im Gesetz steht, ${ }^{37)}$

Wenn der Mensch, der das Haus baute, im Recht ist, dann wird man zu dem Menschen sagen, der ihn behindert hat: »Lege einen Eid ab ( $\mathbf{x}+\mathbf{V I I}, \mathbf{2 2})$ über die Sachen, von denen der Mensch sagt: >Sie sind mir verlorengegangen wegen des ihn Behinderns, das du gemacht hast.«

Siehe den Eid, den man ${ }^{38)}$ ablegt: »Soundso viel Geld, von dem du sagst: >Es ist mir verlorengegangen wegen $(\mathbf{x}+\mathbf{V I I}, \mathbf{2 3})$ des dich Behinderns, das ich gemacht habe bei Errich-

36. Vielleicht sind die Dokumente gemeint.

37. Mit dem Abschnitt $x+V I I, 19-32$ beschäftigt sich E. Seidl: ders, Das private Verbot eines Baues nach dem Rechtsbuch von Hermupolis, in: H. J. Thissen/K. Th. Zauzich (Hg.), Grammata Demotika, FS E. Lüddeckens, Würzburg 1984, 189-192.

38. Bislang wurde an der leicht beschädigten Stelle $n t i w=f$ gelesen, mir scheint vielmehr $n t i w=w$ dazustehen, so daß auch die Gesamtaussage allgemeiner bleibt. Vgl. ähnliche Formeln zur Präsentation von Textvorlagen mit $n t$ i $w=w$ in $\mathrm{x}+\mathrm{II}, 12 ; \mathrm{x}+\mathrm{III}, 23$ und 30 . 
tung deines Hauses. ${ }^{39}$ ) Es ist dir nicht verlorengegangen wegen des Behinderns deines Hauses, das ich gemacht habe. « Wenn er keinen Eid leistet, dann wird man den Menschen, ( $\mathbf{x}+\mathbf{V I I}, \mathbf{2 4})$ der klagt, den Eid leisten lassen: „Soundso viel Geld ist mir verlorengegangen wegen des Behinderns meines Hauses, das du gemacht hast.« Wenn er einen Eid leistet, wird man ihn das Geld dem Kläger geben lassen.

$(\mathbf{x}+\mathbf{V I I}, \mathbf{2 5})$ Es gibt einen Menschen und er behindert ein Haus, um zu verhindern, daß man es baut, obwohl es nicht seines ist. (Im) Namen eines anderen Menschen behindert er es, obwohl der Mensch, den er behinderte, nicht $(\mathbf{x}+\mathbf{V I I}, \mathbf{2 6})$ im nämlichen Ort mit ihm ist. Wenn der Mensch, [der behindert wurde,] um zu verhindern, daß 〈er〉 baut, klagt gegen den Menschen, der behindert hat, wird man ihn fragen. Wenn er sagt »Dieses Haus, es gehört dem Soundso, Sohn des Soundso. ( $\mathbf{x}+\mathbf{V I I}, \mathbf{2 7})$ Er ist nicht (an) diesem Ort. Erst wenn sein Herr kommt, behindere ich ihn, um zu verhindern, daß er $\langle$ es〉 baut«, dann wird man sagen zu dem Menschen, der behindert hat: »Der Mensch, in dessen Namen du (x+VII, 28) dieses Haus behindert hast, bringe inn innerhalb von 30 Tagen.« Wenn 30 Tage vorübergegangen sind, ohne daß er den nämlichen Menschen gebracht hat, wird man den Menschen, der behindert wurde, bauen lassen. Man wird ihn (den Hinderer) bestrafen gemäß dem, was oben geschrieben steht. ( $x+\mathbf{v i l}$, 29) Wenn ein Mensch ein Haus behindert, um zu verhindern, daß es gebaut werde, und er sagt »Du, der gebaut hat, baue nicht«, und wenn er dennoch baut, und wenn er klagt beim Wesir ${ }^{40)}$ ) $\gg l c h$ habe zu dem, der baut, gesagt: $(\mathbf{x}+\mathbf{V I I}, \mathbf{3 0})>$ Baue das Haus nicht.< Er hat nicht auf mich gehört. Er hat widerrechtlich gebaut«, dann wird man den, der gebaut hat, befragen. Wenn er sagt »Er hat mich nicht gehindert«, dann wird man zu ihm sagen: » Lege einen Eid ab: ( $\mathbf{x}+\mathbf{V I I}, \mathbf{3 1})>$ Der Soundso, Sohn des Soundso, hat nicht gesagt: »Baue das Haus nicht.«« Wenn er nicht schwört, dann wird man ihn schlagen mit 50 (?) Stocksch[lägen]. Man baut das Haus nicht, ( $x+\mathrm{VII}, 31)$ bis seine Entscheidung protokolliert ist. $(\mathbf{x}+\mathbf{V} \mathbf{I I}, \mathbf{1})$ Wenn ein Mensch (den Bau) eines Hauses behindert, sagend »Sie geben hinzu [von der] Straße Pharaos ${ }^{41}$ «, dann betrachten [die Richter das Haus ...〈ca. $7 \mathrm{~cm}\rangle \ldots . .].(\mathbf{x}+\mathbf{V I I I}, \mathbf{2})$ welches er zu seinem Haus hinzugegeben hat. Das, was sie finden werden, daß er es von der Straße weggenommen hat $[\ldots\langle\mathrm{ca} .11 \mathrm{~cm}\rangle \ldots]$ $(\mathbf{x}+\mathbf{V I I I}, \mathbf{3})$ Wenn ein Mensch (den Bau) eines Hauses behindert, indem es seinem Haus nahekommt, sagend $[\gg \ldots\langle 12 \mathrm{~cm}\rangle \ldots]^{(x+V I I I, 4)}$ mein Haus $[\ldots \ldots \ldots \ldots \ldots \ll$,$] läßt man es$ ihn nicht bauen, wenn er nicht das Haus einen stehenden (?) Ziegel (weit) daneben gestellt hat $[\ldots\langle\mathrm{ca} .5 \mathrm{~cm}\rangle \ldots]$

$(\mathbf{x}+\mathbf{V I I I}, \mathbf{5})$ Wenn ein Mensch klagt gegen einen Menschen: $\gg$ Er hat den Weg zu meinem Haus weggenommen, er hat ihn zu seinem Haus hinzugefügt«, dann wird man das nämliche Haus betrachten. Wenn $[\ldots \ldots \ldots \ldots \ldots \ldots . . . . .,(x+V I I, 6)$ Fundament auf dem nämlichen Weg (und) es dem Menschen gehört, ${ }^{42)}$ der das Haus baut, dann läßt man

39. Der Schreiber hat hier die Personalpronomina verwechselt. Tatsächlich meinte er: "Es ist mir verlorengegangen wegen des mich Behinderns, das du gemacht hast bei Errichtung meines Hauses."

40. S. S. L. Lippert: $t_{5}$.ty statt $t b-m-m s \check{s}^{e}$ - Neues zum Wesir im Demotischen, in: ZÄS 130 (2003) 88-97.

41. Gemeint ist eine öffentliche Straße.

42. Dem folgenden Zusammenhang nach müßte hier gestanden haben: $i w\left[=f h p r b n-i w t_{5} y=f\right]$

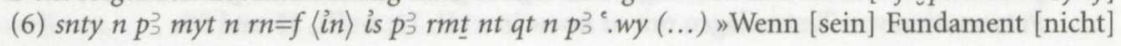


ihn auf dem Fun[dament] bauen. Wenn [sein] (x+vIII, 7) Fundament auf dem nämlichen Weg ist, dann läßt man ihn nicht bauen.

Wenn ein Mensch klagt gegen einen Menschen wegen einer Mauer $(\mathbf{x}+\mathbf{V I I I}, \mathbf{8})$ »Die Mauer [meines Hauses] ist es«, und der Beklagte sagt »Die Mauer meines Hauses ist es«, dann wird man die nämliche Mauer [des Hauses] betrachten, $(\mathbf{x}+\mathbf{V I I I}, \mathbf{9})$ dem sie nahekommt. Man wird es dem geben, dem sie näher ist.

Wenn ein Mensch klagt gegen einen Menschen wegen einer Mauer »Sie gehört zu meinem Haus « (x+VIII, 10) und wenn der Beklagte sagt »[Sie] gehört ihm nicht. Sie gehört nicht zu seinem Haus. Sie ist meine, sie gehört zu meinem Haus.« Wenn das Haus $(\mathbf{x}+\mathbf{V I I I}, \mathbf{1 1})$ in dem Ort ist, in welchem die Richter sind, dann betrachten es die Richter. Der Mensch, von dem die Richter der Ansicht sind, daß $(\mathbf{x + V I I I , 1 2 )}$ sie zu seinem Haus gehört, man wird einen Beschluß auf Anordnung der Richter erlassen, um sie ihm zu geben: »Beschluß: Die Mauer, von der A sagt >Sie ist meines, während B sagt $(\mathbf{x}+\mathbf{V I I I}, \mathbf{1 3})>$ Sie ist meines, die Richter haben sie betrachtet und sie sagen: >Sie gehört dem Soundso.« Man gibt sie dem, von dem gesagt wurde, daß sie seine sei. Und man läßt seinen Prozeßgegner ihm eine Abstandsschrift darüber ( $\mathbf{x}+\mathbf{V I I I}, \mathbf{1 4})$ schreiben. Wenn aber das Haus nicht in dem Ort ist, an dem die Richter sind, einen Beweis über die

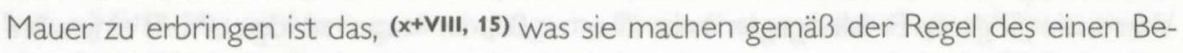
weis Erbringens, das man machen wird. ${ }^{43)}$

Wenn ein Mensch klagt gegen einen Menschen wegen einer Straße »Sie gehört zu meinem (x+viII, 16) Haus«, und wenn der Beklagte sagt »Sie gehört zu meinem Haus«, dann wird man mit ihnen verfahren gemäß dem Gesetz, das oben geschrieben steht.

Wenn ein Mensch klagt $(\mathbf{x}+\mathbf{V I I I}, \mathbf{1 7})$ gegen einen Menschen »Die Regenrinne seines Hauses, sie ergießt Wasser auf mein Haus«, dann wird man die Regenrinne betrachten, indem man Wasser auf sie gibt. ( $\mathbf{x}+\mathbf{V} \mathbf{I I}, \mathbf{1 8})$ Wenn das Wasser [das Haus des] Klägers erreicht, dann wird man die Entwässerung abschneiden, bis man verhindert, daß Wasser sein Haus erreicht.

$(\mathbf{x}+\mathbf{V I I I}, \mathbf{1 9})$ Wenn ein Mensch klagt gegen einen Menschen »Er hat die Tür seines Hauses zu meinem Grundstück hin geöffnet«, und wenn der Beklagte keinen Anspruch auf (x+VIII, 20) das [Grundstück] hat, dann wird man die Tür des Hauses ganz verschließen.

Wenn ein Mensch klagt gegen einen Menschen: $) E r$ hat gegraben neben $(\mathbf{x}+\mathbf{V I I I}, \mathbf{2 1})$ meinem Haus. Er hat mein Haus einstürzen lassen«, dann wird man den Beklagten befragen. Wenn er sagt: »lch habe es nicht getan, um sein Haus einstürzen zu lassen, sondern allein, (x+viII, 22) um [das] Fundament meines Hauses, [das] ich bauen werde, zu graben ${ }^{44}$ « , und wenn er ihm schwört, wird man ihn fern sein lassen von ihm. Wenn er nicht schwört, wird man schwören lassen $(\mathbf{x}+\mathbf{V I I I}, \mathbf{2 3})$ den [Kläger:] »Mein Haus ist eingestürzt. Der und der Besitz ist mir verloren gegangen wegen meines Hauses, das eingestürzt ist. « Wenn er schwört, dann wird man ihn geben lassen ( $\mathbf{x}+\mathbf{V I I I}, \mathbf{2 4})$ den Besitz, [den] der Mensch, dessen Haus eingestürzt ist, [verloren hat.]

auf dem nämlichen Weg ist (und) es dem Menschen gehört, der baut (...)«. Allerdings stehen dem Platzprobleme und nicht dazu passende Zeichenreste entgegen.

43. Ab diesem Satz bis Zeile 21 liegt wieder ein Fragment der griechischen Übersetzung vor (vgl. P. W. Pestman, in: ders. [éd.], Textes et études de papyrologie grecque, démotique et copte, 116-143).

44. Hier endet das zweite Fragment der griechischen Übersetzung. 
Wenn der Mensch, der das Fundament gegraben hat, klagt gegen den Menschen, dessen Haus eingestürzt ist: $(\mathbf{x}+\mathbf{V} \mathbf{I I}, \mathbf{2 5})$ »)Möge er den Schutt seines Hauses [ent]fernen, das in das Fundament meines Hauses gefallen ist«, dann wird man zu ihm sagen: »Lege einen Eid ab: >Schutt ( $\mathbf{x}+\mathbf{v}$ III, 26) meines Hauses ist nicht auf das Haus des Soundso gefallen.« Wenn er keinen Eid ablegt, dann wird man ihm sagen: »Entferne den Schutt deines Hauses, das gefallen ist ( $\mathbf{x}+\mathbf{V I I I}, \mathbf{2 7})$ auf das [nämliche] Haus.《

Wenn ein Mensch klagt gegen einen Menschen »Er hat mich aus meinem Haus geworfen, während ich darin war, um gegen ihn zu klagen.« (x+viII, 28) dann werden die Rich[t]er zum Wesir sagen: »Schicke einen Menschen hinaus zusammen mit dem Soundso, Sohn des Soundso. Lasse sie es in Erfahrung bringen, ob er in dem Haus wohnte ( $\mathbf{x}+\mathbf{V} \mathbf{I I}, \mathbf{2 9})$ bis zum Tag (seines Hinauswurfs). « Wenn man es herausfindet, daß er in dem Haus wohnte bis zum Tag (seines Hinauswurfs), wird der Wesir ihn in nämlichem Haus wohnen lassen.

$(\mathbf{x}+\mathbf{V I I I}, \mathbf{3 0})$ Wenn ein Mensch [gestorben ist], indem er Ackerland, einen Garten, 〈Häuser), Tempelanteile oder einen Diener hat und indem er Nachkommen hat, aber er keine Teilungsschrift geschrieben hat für seine Kinder, als er lebte: Sein ältester Sohn ist der, der ergreift $(\mathbf{x}+\mathbf{V I I I}, \mathbf{3 1})$ seinen Besitz.

Wenn die jüngeren Brüder gegen ihren ältesten Bruder klagen »Möge er uns einen Anteil an dem Besitz unseres Vaters geben«, dann wird der älteste Bruder die Liste ( $\mathbf{x}+\mathbf{V I I I}$, 32) seiner jüngeren Brüder aufschreiben - der Kinder seines Vaters, derer, die leben, und derer, die gestorben sind, bevor ihr Vater gestorben ist, ebenso des ältesten Bruders. Und man wird ihm den Anteil 〈geben〉, (x+VIII, 33) welchen er wünscht, von den Äcker[n, den Gärten] und den Häusern. 〈Das,〉 was angemessen ist, um es ihm zu geben, man gibt es ihm: Die Diener und die Dokumente über das Saatgut und die Güter, $(\mathbf{x + 1} \mathbf{x}, \mathbf{1})$ die seinem Vater gehörten, die (aber bei anderen) Leuten sind, man gibt es ihm. Außer dem Dokument, welches geschrieben ist auf Anordnung seines Vaters und eines anderen Menschen, man gibt es ihm nicht. Den Rest des Besitzes von dem, was [er besessen hat,] $(\mathbf{x}+\mathbf{1} \mathbf{x}, \mathbf{2})$ man teilt es schließlich auf in Teile gemäß der Zahl seiner Kinder. Danach nehmen seine männlichen Kinder (ihren) Anteil [ge]mäß ihrer 〈Geburts) reihenfolge, und seine weiblichen Kinder nach ihnen $(x+1 x, 3)$ gemäß ihrer Geburtsreihenfolge. Wenn ein Mensch unter ihnen ist, der gestorben ist, ${ }^{45)}$ nachdem sein Vater gestorben ist, indem er ohne Sohn ist, nimmt der älteste Sohn (des Vaters) ${ }^{46)}$ seinen Anteil. \{Der Mensch unter ihnen, der $(\mathbf{x}+\mathbf{1} \mathbf{x}, \mathbf{4})$ gestorben ist, nachdem ihm 〈sein) Anteil gegeben wurde, der älteste Sohn ist der, der seinen Anteil nimmt. ${ }^{47)}$ Der Mensch un-

45. Der von S. Grunert (Der Kodex Hermopolis und ausgewählte private Rechtsurkunden aus dem ptolemäischen Ägypten, Leipzig 1982, 84) hier konjizierte Freitod eines Erben ist ohne Grundlage und Notwendigkeit, denn $r m w . t=f$ ist nicht das Partizip der Vergangenheit, weil das Bezugswort indeterminiert ist, sondern ein Circumstantialsatz $r(i w)+s d m=f$.

46. K. Donker van Heel (in: aaO 97) möchte mit P. W. Pestman hier $p_{3}^{3} s^{\circ}{ }^{\circ}$ »der älteste Sohn« zu $p 3 n{ }_{3}$ »der älteste Bruder « emendieren. Dieser Eingriff ist nicht unbedingt notwendig. In $\mathrm{x}+\mathrm{VIII}, 30$ wurde der als Haupterbe Agierende ebenfalls als der älteste Sohn des Verstorbenen bezeichnet.

47. Der folgende Satz verlöre seinen Sinn, wenn dieser erste Satz hier nicht fehlerhaft wäre, weil er allgemeiner ist als der folgende. Es ist vielmehr wahrscheinlich, daß beim Kopieren der Schreiber den Zusatz der Kinderlosigkeit des jüngeren Bruders vergaß, den Fehler bemerkte und den Satz nochmals wiederholte. 
ter ihnen, der gestorben ist, nachdem ihm sein Anteil gegeben (wurde,) indem er keinen [Sohn] hat, der älteste Sohn (seines Vaters) ${ }^{48)}$ ist der, der seinen Anteil nimmt. $(\mathbf{x}+\mathbf{1} \mathbf{x}, \mathbf{5})$ Wenn der jüngere Bruder klagt: $\gg D i e$ Kinder, von denen unser ältester Bruder sagt >Sie sind von unserem Vater<, sie sind nicht Nachkommen [unseres Vaters ....... die Kin]der, von denen der jüngste Bruder [sagt] $(\mathbf{x + 1 \mathbf { x } , 6 )})$ Sie sind nicht von unserem Vater«, man läßt den ältesten Bruder über sie schwören: »Die Kinder, von denen ich gesagt habe >Sie sind von [unserem Vater<, sie sind Nachkommen unseres Vaters. Ich habe nicht ge]logen darüber« $(\mathbf{x}+\mathbf{1} \mathbf{x}, \mathbf{7})$ Man läßt ihn sagen ihre Namen und gleichzeitig (den Namen) ihrer Mutter.

Wortlaut des Eides, den man ihn ablegen läßt: »Der Soundso, [seine Mutter ist die Soundso. Der Soundso, seine Mutter ist die Soundso. Der Soundso, seine Mutter ist die Soundso.] ${ }^{49)}$ Sie sind Nachkommen unseres Vaters. $(\mathbf{x}+\mathbf{1 x}, \mathbf{8})$ Sie sind gestorben, bevor ihr Vater gestorben ist«, der, über den er nicht schwört, man gibt ihm keinen Anteil.

[Der, über den er schwört,] man gibt ihm einen Anteil. $(\mathbf{x}+\mathbf{1 x}, \mathbf{9})$ Wenn eine Tochter diejenige ist, die ihm geboren wurde, gibt man ihr den nämlichen Anteil.

Wenn ein Mensch stirbt, [dessen Besitztümer (o. ä.) sich bei seinem] ältesten [Sohn be-

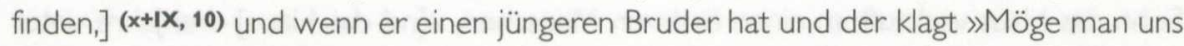
einen Anteil an dem Besitz unseres Vaters geben lassen«, dann wird man aufteilen [... $\langle$ ca. $9 \mathrm{~cm}\rangle \ldots]^{(\mathbf{x}+\mathbf{1 x}, \mathbf{1 1})}$ gemäß der Zahl seiner Kinder, und man gibt einen Anteil zusätzlich dem ältesten Bruder, um zwei Anteile voll zu machen. Wenn er einen [jüngeren] Bruder hat $[\ldots\langle$ ca. $9 \mathrm{~cm}\rangle \ldots]$ Man gibt $(\mathbf{x + 1 x}, \mathbf{1 2}) \mathrm{ihm}$ den Rest des Besitzes [... $\langle\mathrm{ca}$. $10 \mathrm{~cm}\rangle \ldots]$ mit ihnen. Man wird aufteilen $[\ldots\langle\mathrm{ca} .5 \mathrm{~cm}\rangle \ldots$ Wenn $]\{(\mathbf{x}+\mathbf{1 x}, \mathbf{1 3})\}{ }^{50)}(\mathbf{x}+\mathbf{1 x}$, 14) der Mensch keinen Besitz hat außer einem einzigen Haus $[\ldots\langle\mathrm{ca} .5 \mathrm{~cm}\rangle \ldots]$ Dann teilt man nicht [das Haus in Anteile.

Wenn] ein Mensch stirbt, indem er keinen $(\mathbf{x + 1 x}, \mathbf{1 5})$ männlichen Nachkommen hat, sondern weibliche Nachkommen, teilt man seinen Besitz in Anteile [gemäß der Anzahl seiner] Töchter ....... [.........die] Töchter, die er hat. Außer $(\mathbf{x}+\mathbf{1} \mathbf{x}, \mathbf{1 6})$ dem zusätzlichen Anteil eines Kindes, [wird man] es seiner ältesten Tochter geben [... $\langle$ ca. $2 \mathrm{~cm}\rangle \ldots]$, um zwei Anteile voll zu machen. ${ }^{51)}$ Wenn die älteste Tochter sagt52) »Es gab andere Kind[er meines Vaters,] sie sind [tot.] Veranlasse, daß mir ihre Anteil[e] gegeben werden «, $(\mathbf{x}+\mathbf{1} \mathbf{x}$, 17) gibt man ihr nicht [die Anteile] ihrer Schwestern, die tot sind.

Wenn ein Mensch stirbt, indem er Besitz in der Hand seines jüngeren Sohnes hat, und

48. Vgl. die vorhergehende Anmerkung, die auch für diese Stelle gilt.

49. K. Donker van Heel (in: aaO 99) rekonstruiert hier anders, indem er insgesamt nur zwei Söhne mit Mutter angibt, aber dazu noch jeweils den Vatersnamen der Söhne: $p_{3}^{3} m n s_{\supset} p_{\supset}^{3}$ $m n m w . t=f t_{5} m n$ "Der Soundso, Sohn des Soundso, seine Mutter ist die Soundso." Doch der Vater ist ja nach den Angaben des ältesten Bruders sicher, und es heißt zuvor ausdrücklich, daß der älteste Sohn die Namen der Kinder und ihrer Mutter nennen soll. Ich glaube daher, daß stellvertretend dreimal nur $p$ s $m n m w . t=f t_{3} m n$ geschrieben stand.

50. In den Tafeln der editio princeps ist Zeile 12 irrtümlich als zwei Zeilen aufgefaßt und gezählt (vgl. K. Donker van Heel, aaO 100).

51. Eine Ergänzung nach $\mathrm{x}+\mathrm{IX}, 11$ ist nach verfügbarem Platz und Zusammenhang nicht möglich.

52. Das tatsächlich dastehende $b w$-ir $=w$ tỉ d $t_{5}$ s šr.t $t^{e}$.t $»$ man läßt die älteste Tochter nicht sagen « ist zu $i w t_{5} s r^{r} t^{e}{ }_{3} . t \underline{d}$ zu korrigieren (vgl. K. Donker van Heel, aaO 101). 
wenn der älteste Sohn gegen ihn darüber klagt, und wenn $(\mathbf{x}+\mathbf{1} \mathbf{x}, \mathbf{1 8})$ der jüngere Sohn sagt »Der nämliche Besitz, [mein Vater] ist es, der ihn mir gegeben hat, sagend: >Nimm ihn für dich!««, dann wird man ihn schwören lassen: »Mein Vater ist der, der [mir diesen] Besitz gegeben hat, sagend: >Nimm ihn dir « $(\mathbf{x}+\mathbf{1} \mathbf{x}, \mathbf{1 9})\langle$ Wenn er schwört, $\rangle{ }^{53)}$ dann gibt man [es] nicht seinem älteren Bruder. Wenn er nicht schwört, dann gibt man den Besitz seinem älteren Bruder, indem man ihn schreiben läßt über den Besitz [seines Vaters. Wenn es ein Mensch ist, indem ( $\mathbf{x}+\mathbf{1} \mathbf{x}, \mathbf{2 0})$ er keinen Besitz hat außer einem Haus, [ $\langle$ wird man das] Haus [teilen in] Anteile gemäß der Zahl der Kinder von ihm, die, die leben, und die, die [gestorben sind vor dem Tod ihres] Vaters - in Anwesenheit des ältesten Sohnes. $(\mathbf{x}+\mathbf{1} \mathbf{x}, \mathbf{2 1})$ Und man wird einen zusätzlichen Anteil dem ältesten Sohn geben, um [voll zu machen] zwei [Anteile.] Und man wird einem jeden einzelnen von dem Rest der Nachkommen geben, welche er hat, gemäß diesem, was oben geschrieben ist.

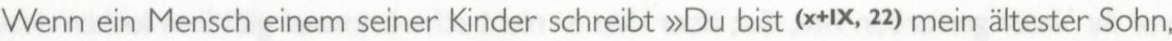
ich habe dir alles gegeben, was ich habe«, wenn der Mensch stirbt, ohne daß er 〈für einen anderen geschrieben hat, können [seine] Brüder nicht gegen ihn klagen wegen

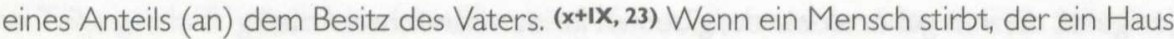
hat, und er nicht darüber für einen Sohn von ihm geschrieben hat, wenn der älteste Sohn das Haus verkauft an einen anderen Menschen, $\mathrm{u}$ [nd das Haus] drei Jahre unter dem Menschen ist, dem er es gegeben hat, $(\mathbf{x + 1 \mathbf { x }}, \mathbf{2 4 )}$ indem keiner gegen ihn geklagt hat, so daß [das] Haus für inn (juristisch) rein ist, und wenn seine jüngeren Brüder ${ }^{54)}$ gegen ihren ältesten Bruder klagen: »Möge man uns Anteil an dem Haus unseres Vaters ge-

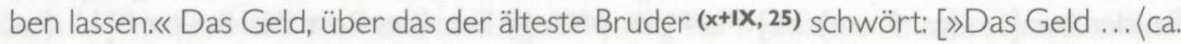
$2 \mathrm{~cm}\rangle$.... Preis dieses Hauses. Ich habe nicht falsch darin gehandelt.«55) Und die Summe des Geldes, über die er einen Eid ablegt, man verlangt sie $(\mathbf{x}+\mathbf{1} \mathbf{x}, \mathbf{2 6})$ von ihm. Und man teilt sie (sc. die Summe) [in Anteile ge]mäß [der Zahl] der jüngeren Brüder.

Wenn ein Mensch sein Haus verkauft (an) einen anderen Menschen, und das Haus 36 Monate unter ihm ist, und man ihm ( $\mathbf{x + 1 x}, \mathbf{2 7})$ einen Pachtvertrag darüber macht jährlich, indem die Pachtverträge $[\ldots \ldots \ldots \ldots \ldots \ldots \ldots . . . . . . . .$. ohne daß ein Mensch geklagt hat dagegen und ohne daß man einen öffentlichen Protest gegen ihn gemacht hat, dann gehört das Haus dem, der es gekauft hat, außer bei $(\mathbf{x}+\mathbf{1 x}, \mathbf{2 8})$ dem Haus, weswegen ein Mensch klagt [gegen einen Menschen:] »Es [gehört] nicht ${ }^{56)}$ dem, der [es] dir [ver]kauft hat. « Wenn es ein Haus ist, welches einem Menschen eingeschrieben ist im (x+1) 29) Grundamt, 57) und ein anderer Mensch es ver[kauft] einem Menschen, der Mensch, dem es verkauft wurde, man läßt ihn nicht sagen: »Es ist für mich (juristisch) rein.«

53. Statt des irrtümlich geschriebenen $i w=w$ t ${ }^{\circ}{ }^{c} r q=f$ »man läßt ihn schwören « ist $i w=f^{c} r q$ zu lesen (vgl. K. Donker van Heel, aaO 103).

54. Bei K. Donker van Heel (in: aaO 105) wurde anders gelesen ( $p s n h m\{. w\} » l$ frère cadet «), doch der folgende Zusammenhang macht deutlich, daß es sich um einen Plural handeln muß, weshalb das ähnlich aussehende $p$ tatsächlich $n \lesssim y=f$ ist und auch der Plural von $\mathrm{hm}$ (hm.w) ernstzunehmen ist. Ähnlich S. Grunert (in: aaO 87) freilich auch nicht ganz korrekt, weil er »die jüngeren Brüder « übersetzt.

55. K. Donker van Heel (in: aaO 105) transliteriert hier $m d^{2} d \underline{d}$ n.im $=w \ldots t$. ip (...), doch ist $m t$ $n^{i} d n$-im $=w$ mtw $t_{5}$ ip (...) deutlich geschrieben.

56. $b n-i w=f$ nach $d$ wurde von $\mathrm{K}$. Donker van Heel (in: aaO 105) nicht erkannt, ist aber trotz Beschädigung zu sehen und auch wegen der Postnegation in zu erwarten.

57. Wörtlich "Ort der Urkunden«. 


\section{Rechtskodex von Hermupolis}

Der Mensch, dem zuerst eine Tochter $(\mathbf{x}+\mathbf{1 x}, \mathbf{3 0})$ geboren wird, und dem danach ein Sohn geboren wird: Die Söhne sind die, die ihm einen ältesten Sohn ergeben. ${ }^{58)}$

Es gibt die Häuser, die erbaut werden aus Stein oder Ziegel, $(\mathbf{x}+\mathbf{I} \mathbf{x}, \mathbf{3 1})$ um darin einen Menschen zu bestatten. Wenn keiner darin bestattet wurde, dann hat ihr Herr das Recht, sie zu verkaufen an einen anderen Menschen. Wenn man in ihnen bestattet, (x+1x, 32) ihr Herr hat nicht das Recht, sie zu verkaufen.

Keiner kann sagen: »Der Besitz ist meiner, denn er gehörte meinem Vater« Außer dem

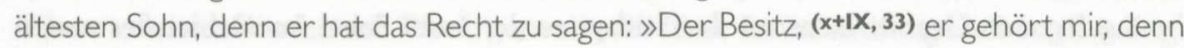
er gehörte meinem Vater.«

58. D. h. nicht das erstgeborene Kind ist Haupterbe, sondern der erstgeborene Sohn, auch wenn er eine ältere Schwester hat. 